\title{
Characterizations and Infinite Divisibility of Certain Recently Introduced Distributions IV
}

\author{
G.G. Hamedani ${ }^{1}$ \\ ${ }^{1}$ Department of Mathematics, Statistics and Computer Science, Marquette University, USA \\ Correspondence: Author, Department of Mathematics, Statistics and Computer Science, Marquette University, Milwau- \\ kee, WI 53201-1881, USA. \\ Received: January 31, 2018 Accepted: February 22, 2018 Online Published: April 18, 2018 \\ doi:10.5539/ijsp.v7n3p36 \\ URL: https://doi.org/10.5539/ijsp.v7n3p36

\begin{abstract}
Certain characterizations of recently proposed univariate continuous distributions are presented in different directions. This work contains a good number of reintroduced distributions and may serve as a source of preventing the reinvention and/or duplication of the existing distributions in the future.
\end{abstract}

Keywords: Univariate continuous distributions, Hazard function, Reverse hazard function, Characterizations

\section{Introduction}

This work is a continuation of our previous works (Hamedani and Safavimanesh, 2016), (Hamedani, 2017) and (Hamedani, 2018) on characterizations and infinite divisibility of distributions introduced in 2016-2018. The current work and our previous published papers mentioned above may serve as a source of preventing the reinvention and/or duplication of the existing distributions in the future. As pointed out in our papers, a good number of proposed distributions have already been introduced in the literature. We believe the authors should do a detailed literature search before spending time on the already existing distributions. In designing a stochastic model for a particular modeling problem, an investigator will be vitally interested to know if their model fits the requirements of a specific underlying probability distribution. To this end, the investigator will rely on the characterizations of the selected distribution. Thus, the problem of characterizing a distribution is an important problem in various fields and has recently attracted the attention of many researchers. Consequently, various characterization results have been reported in the literature. These characterizations have been established in different directions. This work deals with various characterizations of Erlang-Lindley (EL) distribution of Abd El-Monsef et al.; Exponentiated Transmuted Weibull Geometric (ETWG) distribution of Fattah et al.; Transmuted Generalized Exponential (TGE) of Khan et al.; Truncated Inverted Generalized Exponential (TIGE) distribution of Genç; Transmuted Generalized Inverted Exponential (TGIE) distribution of Elbatal; Kumaraswamy GEV (KumGEV) distribution of Eljabari and Nadarajah; Generalized Exponential-G (GEG) distribution of Maurya et al.; Type II Odd Lindley Exponential (TIIOLE) distribution of Korkmaz and Yousof; Burr-X Exponentiated Fréchet (BXEF) distribution of Zayed and Butt; Exponentiated Lomax Geometric (ELG) distribution of Hassan and Abd-Allah; Exponential Pareto Power Series (EPPS) distribution of Elbatal et al.; Transmuted Weibull Fréchet (TWFr) distribution of Ahsan ul Haq et al.; Extended Burr XII (EBXII) distribution of Abouelmagd et al.; Generalized Weibull Burr XII (GWBXII) distribution of Maksaei and Altun; Burr Type XII (BTXII) distribution of Kumar; Gamma Burr XII (GBXII) of Guerra et al.; Generalized Marshall-Olkin-Kumaraswamy-G (GMOKw-G) family of distributions of Chakraborty and Handique; Weibull Burr X (WBX) distribution of Ibrahim et al.; Kumaraswamy Transmuted Pareto (KwTP) distribution of Chhetri et al.; Odd Log-Logistic Logarithmic Generated (OLLL-G) family of distributions of Alizadeh et al.; Marshall-Olkin Extended Inverse Pareto (MOEIP) distribution of Gharib et al.; Marshall-Olkin Extended Inverse Weibull (MOEIW) distribution of Okasha et al.; Upper Truncated Lindley (UTL) distribution of Singh et al.; Transmuted Exponentiated Fréchet (TEF) distribution of Elbatal et al.; Generalized Inverted Generalized Exponential (GIGE) distribution of Oguntunde and Adejumo; Transmuted Rayleigh (TR) distribution of Ahmad et al.; McDonald Quasi Lindley (McQL) distribution of Roozegar and Esfandiyari; Geometric Weibull Poisson (GWP) distribution of Mansour and Abd Elrazik; SS Transformation of Exponential (SSTE) distribution of Kumar et al.; Generalization of the BurrXII-Poisson (GBXIIP) distribution of Muhammad; Log-Logistic Generated Weibull (LLGW) distribution of Abdel-Hamid and Albasuoni; Odd Generalized Exponentiated Linear Failure Rate (OGELFR) distribution of El-Damcese et al.; Transmuted Exponentiated Inverse Rayleigh (TEIR) distribution of Ahsan ul Haq; Transmuted Two-Parameter Lindley (TTL) distribution of Al-Khazaleh et al.; Transmuted Janardan (TJ) distribution of Al-Omari et al.; Exponentiated Generalized Weibull-Gompertz (EGWG) distribution of ElBassiouny et al.; Complementary Exponentiated BurrXII Poisson (CEBXIIP) distribution of Muhammad; Kumaraswamy Power Function (Kw-PF) distribution of Abdul-Moniem; Odd Burr III-G (OBIII-G) family of distributions of Jamal et al.; Odd Generalized Exponential Generalized Linear Exponential (OGE-GLE) distribution of Luguterah and Nasiru; 
Exponentiated Inverse Flexible Weibull Extension (EIFWE) distribution of El-Morshedy et al.; Transmuted Weighted Exponential (TWE) distribution of Dar et al.; Beta Transmuted Pareto (BTP) distribution of Chherti et al.; Generalized Burr-G (GBG) family of distributions of Nasir et al.; Exponentiated Weibull-Power Function (EWPF) distribution of Hassan and Assar; Transmuted Two-Parameter Lindley $\left(\mathrm{TTL}_{1}\right)$ distribution of Kemaloglu and Yilmaz; Marshall-Olkin Log-Logistic Extended Weibull (MOLLEW) distribution of Lepetu et al.; Modified Slash Birnbaum-Saunders (MSBS) distribution of Reyes et al.; Inverse Power Lindley (IPL) distribution of Barco et al.; Weibull Weibull (WW) distribution of Abouelmagd et al.; Beta Generated Kumarswamy-G (BGKw-G) family of distributions of Handique et al.; New Life Model (NLM) of Muhammad; Marshall-Olkin Exponentiated Burr XII (MOEBXII) distribution of Cordeiro et al.; Beta Nadarajah-Haghighi (BNH) distribution of Dias et al.; New Two-Parameter Weibull (NTPW) distribution of Rasekhi et al.; Generalized Half-t (GHT) distribution of Bulut et al.; Gamma Extended-G (GE-G) distribution of Cordeiro et al.; Modified Behrens-Fisher (MB-F) distribution of Nadarajah and Li; Maxwell-Weibull (M-W) distribution of Sharma et al.; Exponentiated Weibull-Exponential (EWE) distribution of Elgarhy et al.; $\alpha$ Logarithmic Transformed $(\alpha$ LT) family of distributions of Dey et al.; Topp-Leone Odd Log-Logistics (TLOLL) family of distributions of Brito et al.; Two-Parameter Maxwell (TP-M) distribution of Dey et al.; Generalized Quadratic Hazard Rate (GQHR) distribution of Sarhan; New FourParameter Weibull (NFPW) distribution of Yousof et al.; Odd Lindley Burr XII (OLBXII) distribution of Abouelmagd et al.; Dagum Poisson (DP) distribution of Oluyede et al.; Burr XII Modified Weibull (BXIIMW) of Mdlongwa et al.; Burr X Pareto (BXP) of Korkmaz et al.; General Class of Flexible Weibull (GCFW) distributions of Park and Park; Power Lomax Poisson (PLP) distribution of Hassan and Nassr; Modified Weibull Poisson (MWP) distribution of Ghorbani et al.; Exponentiated Power Lindley Geometric (EPLG) distribution of Alizadeh et al.; Generalized Modified Weibull Power Series (GMWPS) distribution of Bagheri et al.; Additive Modified Weibull Odd Log-Logistic-G (AMWOLLG) family of distributions of Ghorbani Et al.; Exponentiated Power Lindley Power Series (EPLPS) distribution of Alizadeh et al.; Weibull-R (W-R) family of distributions of Ghosh and Nadarajah; Wrapped Lindley (WL) distribution of Joshi and Jose; Beta Weibull-G family of distributions of Makubate et al.; Transmuted Weibull Regression (TWR) distribution of Granzotto et al.. These characterizations are presented in different directions: (i) based on the ratio of two truncated moments; (ii) in terms of the hazard function; (iii) in terms of the reverse (reversed) hazard function and (iv) based on the conditional expectation of certain function of the random variable. Note that $(i)$ can be employed also when the $c d f$ (cumulative distribution function) does not have a closed form. In defining the above distributions we shall try to employ the same parameter notation as used by the original authors. We follow the same order as listed above.

1) The $c d f$ of EL is given by

$$
F(x ; \theta, k)=1-\frac{1}{\Gamma(k)(1+\theta)^{2}}\left\{\theta \Gamma(k) e^{-\theta x}(1+\theta+\theta x)+(1+\theta) \Gamma(k, \theta x)\right\},
$$

$x \geq 0$, where $\theta>0$ and $k \in \mathbb{N}$ are parameters and $\Gamma(k, x)=\int_{x}^{\infty} w^{k-1} e^{-w} d w$.

2) The $c d f$ of ETWG is given by

$$
F(x ; \alpha, \beta, \sigma, \lambda, p)=\frac{\left[1-e^{-\left(\frac{x}{\sigma}\right) \beta}\right]^{\alpha}\left[1+\lambda e^{-\left(\frac{x}{\sigma}\right) \beta}\right]^{\alpha}}{1-p+p\left[1-e^{-\left(\frac{x}{\sigma}\right) \beta}\right]^{\alpha}\left[1+\lambda e^{-\left(\frac{x}{\sigma}\right) \beta}\right]^{\alpha}}, x \geq 0,
$$

where $\alpha, \beta, \sigma$ are all positive, $|\lambda| \leq 1$ and $p \in(0,1)$ are parameters.

3) The $c d f$ of TGE is given by

$$
F(x ; \alpha, \theta, \lambda)=\left[1-e^{-\theta x}\right]^{\alpha}\left\{1+\lambda-\lambda\left(1-e^{-\theta x}\right)^{\alpha}\right\}, x \geq 0,
$$

where $\alpha, \theta$ both positive and $|\lambda| \leq 1$ are parameters.

Remark 1. The TGE distribution of Khan et al. (2017b) is a special case of TEAW distribution of Nofal et al. (2017), which has already been characterized in Hamedani (2017a). We believe that Khan et al. were not aware of Nofal et al.'s paper.

4) The $c d f$ of TIGE is given by

$$
F(x ; \alpha, \lambda)=E-C\left(1-e^{-\lambda / x}\right)^{\alpha}, \quad 0 \leq B \leq x \leq A<\infty,
$$

where $\alpha, \lambda$ are positive parameters and $E=\left\{\left(1-e^{-\lambda / A}\right)^{\alpha}-\left(1-e^{-\lambda / B}\right)^{\alpha}\right\}^{-1}, C=E\left(1-e^{-\lambda / B}\right)^{\alpha}$. 
Remark 2. The TIGE distribution of Genç (2017) is a special case of INGIW distribution of Khan et al. (2017b), which has already been characterized in Hamedani (2017b). We believe that Genç was not aware of Khan et al.'s paper.

5) The $c d f$ of TGIE is given by

$$
F(x ; \alpha, \theta, \lambda)=\left(1-\left(1-e^{-\theta / x}\right)^{\alpha}\right)\left(1+\lambda\left(1-e^{-\theta / x}\right)^{\alpha}\right), \quad x \geq 0,
$$

where $\alpha, \theta$ both positive and $|\lambda| \leq 1$ are parameters.

Remark 3. The TGIE distribution of Elbatal (2013) is a special case of TNGIW distribution of Khan et al. (2017a), which has already been characterized in Hamedani (2017b).

6) The $c d f$ of KumGEV (WLOG, for $\mu=0, \sigma=1$ ) is given by

$$
F(x ; a, b, \eta)=1-\left[1-\exp \left\{-a(1+\eta x)^{-1 / \eta}\right\}\right]^{b}, \quad x \geq 0,
$$

where $a, b, \eta$ are all positive parameters.

7) The $c d f$ of GEG is given by

$$
F(x ; \alpha)=\frac{\exp \left\{(G(x))^{\alpha}\right\}-1}{e-1}, \quad x \in \mathbb{R},
$$

where $\alpha>0$ is a parameter and $G(x), g(x)$ are $c d f$ and $p d f$ (probability density function) of the baseline distribution.

Remark 4. One may add another parameter to GEG and express (7) as $F(x ; \alpha, \beta)=\frac{\exp \left\{\beta(G(x))^{\alpha}\right\}-1}{e^{\beta}-1}, \quad x \in \mathbb{R}$.

8) The $c d f$ of TIIOLE is given by

$$
F(x ; \lambda)=1-\frac{1+\exp (-\lambda x)}{2 \exp (-\lambda x)} \exp \{1-\exp (\lambda x)\}, \quad x \geq 0,
$$

where $\lambda>0$ is a parameter.

Remark 5. The TIIOLE distribution of Korkmaz and Yousof (2017) is a special case of OL-G distribution of GomezSilva et al. (2017), which has been characterized in Hamedani (2017a).

9) The $c d f$ of BXEF is given by

$$
F(x ; \theta, \lambda, \beta, \alpha)=\left(1-\exp \left\{-\left[\frac{1-\left(1-e^{-\alpha x^{-\beta}}\right)^{\lambda}}{\left(1-e^{-\alpha x^{-\beta}}\right)^{\lambda}}\right]^{2}\right\}\right)^{\theta}, \quad x \geq 0,
$$

where $\theta, \lambda, \beta, \alpha$ are all positive parameters.

Remark 6. The BXEF distribution of Zayed and Butt (2017) is a submodel of BX-G distribution introduced by Yousof et al. (2016), which has been characterized in Hamedani (2017a).

10) The $c d f$ of ELG is given by

$$
F(x ; \alpha, \theta, \lambda, p)=\frac{\left[1-(1+\lambda x)^{-\theta}\right]^{\alpha}}{1-p\left\{1-\left[1-(1+\lambda x)^{-\theta}\right]^{\alpha}\right\}}, \quad x \geq 0,
$$

where $\alpha, \theta, \lambda$ all positive and $p(0<p<1)$ are parameters.

11) The $c d f$ of EPPS is given by

$$
F(x ; \alpha, \theta, \lambda, \mu)=1-\frac{c\left[\theta e^{-\alpha\left(\frac{x}{\mu}\right)^{\lambda}}\right]}{c(\theta)}, \quad x \geq 0,
$$

where $\alpha, \theta, \lambda, \mu$ are all positive parameters and $c(\theta)=\sum_{n=1}^{\infty} a_{n} \theta^{n}, a_{n} \geq 0$. 
Remark 7. The EPPS distribution of Elbatal et al. (2017) is a submodel of RGTLPS distribution introduced by Condino and Domma (2016), which has been characterized in Hamedani (2017a).

12) The $c d f$ of TWFr, for $a=1$, is given by

$$
\begin{aligned}
F(x ; \beta, \gamma, \lambda, 1, b)= & \left(1-\exp \left\{-\left[\frac{e^{-\left(\frac{\gamma}{x}\right)^{\beta}}}{1-e^{-\left(\frac{\gamma}{x}\right)^{\beta}}}\right]^{b}\right\}\right) \times \\
& {\left[1+\lambda \exp \left\{-\left[\frac{e^{-\left(\frac{\gamma}{x}\right)^{\beta}}}{1-e^{-\left(\frac{\gamma}{x}\right)^{\beta}}}\right]^{b}\right\}, x \geq 0,\right.}
\end{aligned}
$$

where $\beta, \gamma, b$ all positive and $\lambda(|\lambda| \leq 1)$ are parameters.

13) The $c d f$ of EBXII is given by

$$
F(x ; \alpha, \beta, v, a, b)=\left(1-\exp \left\{-a\left[\left(1+x^{\alpha}\right)^{\beta}-1\right]^{b}\right\}\right)^{v}, \quad x \geq 0,
$$

where $\alpha, \beta, v, a, b$ are all positive parameters.

Remark 8. The EBXII distribution of Abouelmagd et al. (2017) is a minor extension of FPBXII distribution introduced by Afify et al. (2017), which has been characterized in Hamedani (2017a).

14) The $c d f$ of GWBXII is given, by the authors as

$$
F(x ; \alpha, \beta, s, u, k)=1-\exp \left\{-a\left(-\log \left[1+\left(\frac{x}{s}\right)^{u}\right]^{-k}\right)^{\beta}\right\}, \quad x \geq 0 .
$$

It is easy to see that the $c d f$ can be written as

$$
F(x ; \alpha, \beta, s, u, k)=1-\exp \left\{-a k^{\beta}\left(\log \left[1+\left(\frac{x}{s}\right)^{u}\right]\right)^{\beta}\right\}, x \geq 0 .
$$

and WLOG, we can set $k=1$.

Therefore, the $c d f$ of GWBXII (WLOG, we can set $k=1$ ) is given by

$$
F(x ; \alpha, \beta, s, u, 1)=1-\exp \left\{-a\left(\log \left[1+\left(\frac{x}{s}\right)^{u}\right]\right)^{\beta}\right\}, \quad x \geq 0,
$$

where $\alpha, \beta, s, u$ are all positive parameters.

15) The $c d f$ of BTXII is given by

$$
F(x ; \alpha, \beta)=1-\left(1+x^{\alpha}\right)^{-\beta}, x \geq 0,
$$

where $\alpha, \beta$ are all positive parameters.

Remark 9. The BTXII distribution of Kumar (2017) is a special submodel of TBTXII distribution introduced by AlKhazaleh (2016), which has been characterized in Hamedani (2017a).

16) The $c d f$ of GBXII is given by

$$
F(x ; a, c, d, s)=\frac{\gamma\left(a, d \log \left[1+\left(\frac{x}{s}\right)^{c}\right]\right)}{\Gamma(a)}, x \geq 0,
$$

where $a, c, d, s$ are all positive parameters and $\gamma(a, z)=\int_{0}^{z} t^{a-1} e^{-t} d t$. 
Remark 10. The GBXII distribution of Guerra et al. (2017) is a special submodel of G-P distribution introduced by Alzaatreh et al. (2012) as well as a special submodel of GEW distribution of Cordeiro et al.(2016). Both distributions (G-P and GEW) have been characterized in the Research Monograph by Hamedani and Maadooliat (2017).

17) The $c d f$ of GMOKw-G is given by

$$
F(x ; \alpha, \theta, a, b)=1-\left[\frac{\alpha\left[1-G(x)^{a}\right]^{b}}{1-(1-\alpha)\left[1-G(x)^{a}\right]^{b}}\right]^{\theta}, x \in \mathbb{R}
$$

where $\alpha, \theta, a, b$ are all positive parameters and and $G(x)$ is the baseline $c d f$ with corresponding $p d f g(x)$.

Remark 11. A slightly more general case of the GMOKw-G distribution has been characterized in Hamedani (2017a).

18) The $c d f$ of WBX is given by

$$
F(x ; \alpha, \beta, \theta, \lambda)=1-\exp \left\{-\alpha \frac{\left(1-e^{-(\lambda x)^{2}}\right)^{\theta \beta}}{\left[1-\left(1-e^{-(\lambda x)^{2}}\right)^{\theta}\right]^{\beta}}\right\}, x \geq 0
$$

where $\alpha, \beta, \theta, \lambda$ are all positive parameters.

19) The $c d f$ of KwTP is given by

$$
\begin{aligned}
F(x ; \alpha, \theta, \lambda, a, b) & =1-\left[1-G(x ; \alpha, \theta, \lambda)^{a}\right]^{b}, \quad x \in \mathbb{R}, \\
& =1-\left\{1-\left[1-\left(\frac{\theta}{x}\right)^{\alpha}\right]^{a}\left[1+\lambda\left(\frac{\theta}{x}\right)^{\alpha}\right]^{a}\right\}^{b}, x \geq 0,
\end{aligned}
$$

where $\alpha, \theta, a, b$ all positive and $\lambda(|\lambda| \leq 1)$ are parameters.

Remark 12. The distribution $F(x ; \alpha, \theta, \lambda, a, b)=1-\left[1-G(x ; \alpha, \theta, \lambda)^{a}\right]^{b}, x \in \mathbb{R}$, has been considered by several authors before. In fact a more general case was proposed by Mahmoud et al. (2015). For $\lambda=0$, the $c d f$ (37) was taken up by Bourguignon et al. (2013).

20) The $c d f$ of OLLL-G is given by

$$
F(x ; \alpha, \beta, \eta)=\left(\frac{1}{\log (1-\beta)}\right) \log \left[1-\frac{\beta G(x ; \eta)^{\alpha}}{G(x ; \eta)^{\alpha}+\bar{G}(x ; \eta)^{\alpha}}\right], \quad x \in \mathbb{R}
$$

where $\alpha>0,0<\beta<1$ are parameters and $G(x ; \eta)$ is the baseline $c d f$ with corresponding $p d f g(x ; \eta)$.

Remark 13. The Zografos-Balakrishnan Odd Log-Logistic (ZBOLL-G) distribution with $c d f$

$$
F(x ; \alpha, \beta, \tau)=\frac{1}{\Gamma(\beta)} \gamma\left[\beta,-\log \left\{1-\frac{G(x ; \tau)^{\alpha}}{G(x ; \tau)^{\alpha}+\bar{G}(x ; \tau)^{\alpha}}\right\}\right],
$$

where $x \in \mathbb{R}, \alpha>0, \beta>0$ was proposed by Cordeiro et al. (2016). The OLLL-G distribution seems to be a simple variation of ZBOLL-G, which has been characterized in the Research Monograph by Hamedani and Maadooliat (2017).

21) The $c d f$ of MOEIP is given by

$$
F(x ; \alpha, \beta, \delta)=1-\delta\left[1-\left(\frac{x}{x+\beta}\right)^{\alpha}\right]\left[\delta-(\delta-1)\left(\frac{x}{x+\beta}\right)^{\alpha}\right]^{-1},
$$

$x \geq 0$, where $\alpha, \beta, \delta$ are positive parameters.

Remark 14. $\bar{F}(x)$ in equation (6) of Gharib et al. (2017) should be replaced with $F(x)$.

22) The $c d f$ of MOEIW is given by 


$$
F(x ; \alpha, \beta, \theta)=e^{-\alpha x^{-\beta}}\left[\theta-(\theta-1) e^{-\alpha x^{-\beta}}\right]^{-1}, \quad x \geq 0,
$$

where $\alpha, \beta, \theta$ are positive parameters.

Remark 15. The MOEIW distribution was introduced under the name of Marshall-Olkin Fréchet (M-OF) distribution by Krishna et al. (2013). The latter has been characterized in the Research Monograph by Hamedani and Maadooliat (2017). 23) The $c d f$ of UTL is given by

$$
F(x ; \theta, \eta)=\frac{e^{\theta \eta}\left\{1+\theta-[1+\theta(1+x)] e^{-\theta x}\right\}}{(1+\theta)\left(e^{\theta \eta}-1\right)-\theta \eta}, 0 \leq x \leq \eta,
$$

where $\theta, \eta$ are positive parameters.

24) The $c d f$ of TEF, is given by

$$
F(x ; \alpha, \beta, \theta, \lambda)=\left[1-\left(1-e^{-\left(\frac{\theta}{x}\right)^{\beta}}\right)^{\alpha}\right]\left[1+\lambda\left(1-e^{-\left(\frac{\theta}{x}\right)^{\beta}}\right)^{\alpha}\right]
$$

$x \geq 0$, where $\alpha, \beta, \theta$ positive and $\lambda(|\lambda| \leq 1)$ are parameters.

25) The $c d f$ of GIGE is given by

$$
F(x ; \alpha, \gamma, \lambda)=1-\left(1-e^{-\gamma\left(\frac{\lambda}{x}\right)}\right)^{\alpha}, x \geq 0,
$$

where $\alpha, \gamma, \lambda$ are positive parameters.

Remark 16. The GIGE distribution is a special case of, at least two distributions. We mention here, the ExponentiatedExponential Fréchet (EEFr) distribution of Mansoor et al. which has been characterized in the Research Monograph by Hamedani and Maadooliat (2017).

26) The $c d f$ of TR is given by

$$
F(x ; \theta, \lambda)=\left(1-\exp \left(-\frac{x^{2}}{2 \theta^{2}}\right)\right)\left(1+\lambda \exp \left(-\frac{x^{2}}{2 \theta^{2}}\right)\right), \quad x \geq 0
$$

where $\theta, \lambda$ are positive parameters.

Remark 17. The TR distribution is a special case of the Kumaraswamy-Transmuted Exponentiated Modified Weibull (Kw-TEMW) distribution of Al-babtain et al. (Communications in Statist. Theory-Methods, forthcoming) which has been characterized in Hamedani (IJSP, forthcoming).

27) The $c d f$ of McQL is given by

$$
F(x ; a, b, c, \alpha, \theta)=I\left(\left[1-\frac{1+\alpha+\theta x}{\alpha+1} e^{-\theta x}\right]^{c} ; a / c, b\right), x \geq 0
$$

where $a, b, c, \theta$ all positive and $\alpha>-1$ are positive parameters.

Remark 18. For $a=c$ the McQL distribution of Roozegar and Esfandiary (2015) reduces to the Kumaraswamy Quasi Lindley (KQL) distribution of Elbatal and Elgarhy (2013). The KQL distribution has characterized in the Research Monograph by Hamedani and Maadooliat (2017).

28) The $c d f$ of GWP is given by

$$
F(x ; \alpha, \beta, \lambda, \pi)=\frac{(1-\pi)\left[1-\exp \left(-\lambda+\lambda e^{-(\beta x)^{\alpha}}\right)\right]}{1-e^{-\lambda}-\pi+\pi \exp \left(-\lambda+\lambda e^{-(\beta x)^{\alpha}}\right)}, \quad x \geq 0
$$

where $\alpha, \beta, \lambda$ all positive and $\pi(0<\pi<1)$ are parameters.

29) The $c d f$ of SSTE is given by 


$$
F(x ; \theta)=\cos \left(\frac{\pi}{2} e^{-\theta x}\right), \quad x \geq 0,
$$

where $\theta>0$ is a parameter.

30) The $c d f$ of GBXIIP is given by

$$
F(x ; a, \alpha, \beta, \lambda)=\left(\frac{1-\exp \left(\lambda\left(\left(1+x^{\alpha}\right)^{-\beta}-1\right)\right)}{1-e^{-\lambda}}\right)^{a}, x \geq 0
$$

where $a, \alpha, \beta, \lambda$ are positive parameters.

31) The $c d f$ of LLGW is given by

$$
F(x ; \alpha, \beta, \gamma)=1-\left(1+\left(\alpha x^{\beta}\right)\right)^{-1}, x \geq 0,
$$

where $\alpha, \beta, \gamma$ are positive parameters.

Remark 19. The LLGW distribution of Abdel-Hamid and Albasuoni (2016) is a special submodel of TBTXII distribution introduced by Al-Khazaleh (2016), which has been characterized in Hamedani (2017a). Furthermore, LLGW distribution reduces to BTXII distribution for $\gamma=1$, please see Remark 9.

32) The $c d f$ of OGELFR is given by

$$
F(x ; \alpha, \beta, a, b)=\left[1-e^{-\alpha\left(e^{a x+\frac{b}{2} x^{2}}-1\right)}\right]^{\beta}, x \geq 0
$$

where $\alpha, \beta, a, b$ are positive parameters.

Remark 20. The OGELFR distribution of El-Damcese et al. (2016) is a special submodel of Exponentiated Weibull Rayleigh (EWR) distribution of Elgarhy, which has been characterized in Hamedani (2017a).

33) The $c d f$ of TEIR is given by

$$
F(x ; \alpha, \beta, \theta, \lambda)=\left(e^{-\frac{\theta \alpha}{x^{2}}}\right)\left[1+\lambda-\lambda\left(e^{-\frac{\theta \alpha}{x^{2}}}\right)\right], x \geq 0,
$$

where $\alpha, \beta, \theta$ are positive and $\lambda(|\lambda| \leq 1)$ are parameters.

Remark 21. The TEIR distribution of Ahsan ul Haq (2016) is a special submodel of Transmuted Kumaraswamy Exponentiated Inverse Rayleigh (TKEIR) distribution of Badr (2017), which has been characterized in Hamedani (2017a).

34) The $c d f$ of TTL is given by

$$
F(x ; \alpha, \theta, \lambda)=\frac{e^{-2 \theta x}\left[\theta\left(e^{\theta x}-1\right)+\alpha\left(e^{\theta x}-\theta x-1\right)\right] \times}{\left[e^{\theta x}(\alpha+\theta)+\lambda(\alpha+\theta+\alpha \theta x)\right]}, \quad x \geq 0,
$$

where $\theta>0, \alpha>-\theta, \lambda(|\lambda| \leq 1)$ are parameters.

35) The $c d f$ of $\mathrm{TJ}$ is given by

$$
F(x ; \alpha, \theta)=1-\left(1+\frac{\theta \alpha x}{\theta+\alpha^{2}}\right) e^{-\frac{\theta}{\alpha} x}, \quad x \geq 0,
$$

where $\theta>0, \alpha>0$ are parameters.

Remark 22. The TJ distribution of Al-Omari et al. (2017) is similar to the Maxwell Length Biased (MLB) distribution of Saghir et al. (2016) whose $c d f$ is $F(x ; \alpha, \theta)=1-\left(1+\frac{x^{2}}{2 \alpha^{2}}\right) e^{-\frac{x^{2}}{2 \alpha^{2}}}, \quad x \geq 0$. The MLB distribution has been characterized in Hamedani (2017a). 
36) The $c d f$ of EGWG is given by

$$
F(x ; a, b, c, d, \theta)=\left[1-e^{-a x^{b}\left(e^{c^{d}}-1\right)}\right]^{\theta}, \quad x \geq 0,
$$

where $a, b, c, d, \theta$ are all positive parameters.

Remark 23. The EGWG distribution of El-Bassiouny et al. (2017) is the same as the one proposed by El-Damcese et al. (2015). The EGWG distribution is in turn a special case of EWR distribution of Elgarhy (2015) which has been characterized in Hamedani (2017a).

37) The $c d f$ of CEBXIIP is given by

$$
F(x ; \alpha, \beta, \lambda, \theta)=\frac{\exp \left(\lambda\left(1-\left(1+x^{\alpha}\right)^{-\beta}\right)^{\theta}\right)-1}{\exp (\lambda)-1}, x \geq 0,
$$

where $\alpha, \beta, \lambda, \theta$ are all positive parameters.

Remark 24. The CEBXIIP distribution of Muhammad (2017) is a special case of Poisson-G (Po-G) family of distributions of Abouelmagd et al. (2017) if one takes $G(x)=\left(1-\left(1+x^{\alpha}\right)^{-\beta}\right)^{\theta}$ in the formula for the Po-G distribution. The Po-G distribution has been characterized in Hamedani (2018).

38) The $c d f$ of Kw-PF is given by

$$
F(x ; a, b, \lambda, \theta)=1-\left[1-\left(\frac{x}{\lambda}\right)^{a \theta}\right]^{b}, 0 \leq x \leq \lambda,
$$

where $a, b, \lambda, \theta$ are all positive parameters.

39) The $c d f$ of OBIII-G is given by

$$
F(x ; c, \theta)=\left[1+\left(\frac{1-G(x ; \theta)}{G(x ; \theta)}\right)^{c}\right]^{-k}, \quad x \in \mathbb{R}
$$

where $c, k, \theta$ are all positive parameters and $G(x ; \theta)$ is the base $c d f$ with corresponding $p d f g(x ; \theta)$.

Remark 25. The OBIII-G distribution of Jamal et al. (2017) is the same as the one proposed by Arifa et al. (2017), called Modified Burr III G (MBIIIG) distribution, which has been characterized in Hamedani (2017a).

40) The $c d f$ of OGE-GLE is given by

$$
F(x ; \alpha, a, b, c, \lambda)=\left[1-e^{-\lambda\left(e^{\left(a x+\frac{b x^{2}}{2}\right)^{c}}-1\right)}\right]^{\alpha}, x \geq 0,
$$

where $\alpha, a, b, c, \lambda$ are all positive parameters.

Remark 26. The OGE-GLE distribution of Luguterah and Nasiru (2017) is a special case of the distribution of Abdelall (2016), called Odd Generalized Exponential Modified Weibull (OGEMW) distribution, which has been characterized in Hamedani and Safavimanesh (2017).

41) The $c d f$ of EIFWE is given by

$$
F(x ; \alpha, \beta, \lambda)=e^{-\lambda e^{\alpha / x-\beta x}}, \quad x \geq 0,
$$

where $\alpha, \beta, \lambda$ are all positive parameters.

Remark 27. For $\lambda=1$, the EIFWE distribution of El-Morshedy et al. (2017) is the same as the one proposed by the same authors (listed in the different order) in (2015), called Inverse Flexible Weibull Extension (IFWE) distribution. The IFWE has been characterized in a Research Monograph by Hamedani and Maadooliat (2017). 
42) The $c d f$ of TWE is given by

$$
F(x ; \lambda, \omega)=\frac{[\Gamma(\omega+1)-\Gamma(\omega+1, \lambda x)][\Gamma(\omega+1)+\beta \Gamma(\omega+1, \lambda x)]}{[\Gamma(\omega+1)]^{2}}, x \geq 0,
$$

where $\lambda>0, \omega \geq 0$ and $\beta(|\beta| \leq 1)$ are parameters.

43) The $c d f$ of BTP is given by

$$
F\left(x ; \alpha, a, b, x_{0}\right)=\frac{1}{B(a, b)} \int_{0}^{\left[1-\left(\frac{x_{0}}{x}\right)^{\alpha}\right]\left[1+\lambda\left(\frac{x_{0}}{x}\right)^{\alpha}\right]} w^{a-1}(1-w)^{b-1} d w, \quad x \geq x_{0},
$$

where $\alpha, a, b$, and $\lambda(|\lambda| \leq 1)$ are parameters.

Remark 28. For $\lambda=0$, the BTP distribution of Chhetri et al. (2017) reduces to Generalized Beta Exponentiated Pareto (GBEP) distribution of Mead (2014). The GBEP distribution has been characterized in the Research Monograph by Hamedani and Maadootiat (2017).

44) The $c d f$ of GBG is given by

$$
F(x ; c, k, \eta)=1-\left[1+(-\log (\bar{G}(x ; \eta)))^{c}\right]^{-k}, \quad x \in \mathbb{R},
$$

where $c, k$ are positive parameters and $G(x ; \eta)(\bar{G}(x ; \eta)=1-G(x ; \eta))$ is the baseline $c d f$ with the corresponding $p d f$ $g(x ; \eta)$, which may depend on the vector parameter $\eta$.

45) The $c d f$ of EWPF is given by

$$
F(x ; \alpha, \beta, \lambda, \theta, a)=\left[1-\exp \left(-\alpha\left(\frac{x^{\theta}}{\lambda^{\theta}-x^{\theta}}\right)^{\beta}\right)\right]^{a},
$$

$0 \leq x \leq \lambda$, where $\alpha, \beta, \lambda, \theta, a$ are all positive parameters.

Remark 29. For $a=1$, the EWPF distribution of Hassan and Assar (2017) reduces to Weibull Power Function (WPF) distribution of Tahir et al. (2014). The WPF distribution has been characterized in the Research Monograph by Hamedani and Maadootiat (2017).

46) The $c d f$ of $\mathrm{TTL}_{1}$ is given by

$$
F(x ; \alpha, \theta, \lambda)=(1+\lambda)\left[1-\left(\frac{\theta+\alpha+\alpha \theta x}{\theta+\alpha}\right) e^{-\theta x}\right]-\lambda\left[1-\left(\frac{\theta+\alpha+\alpha \theta x}{\theta+\alpha}\right) e^{-\theta x}\right]^{2},
$$

$x \geq 0$, where $\alpha>0, \theta>0, \lambda(|\lambda| \leq 1)$ are parameters.

Remark 30. The TLL 1 distribution of Kemaloglu and Yilmaz (2017) is the same as the TTL distribution of Al-Khazaleh

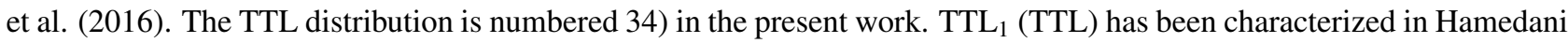
(2017a).

47) The $c d f$ of MOLLEW is given by

$$
F(x ; c, \alpha, \delta, \eta)=1-\frac{\delta\left(1+x^{c}\right)^{-1} e^{-\alpha H(x ; \eta)}}{1-(1-\delta)\left(1+x^{c}\right)^{-1} e^{-\alpha H(x ; \eta)}}, x \geq 0,
$$

where $\alpha>0, \delta>0$ are parameters and $H(x ; \eta)$ is a non-negative differentiable function with $\lim _{x \rightarrow 0^{+}} H(x ; \eta)=0$, $h(x ; \eta)=H^{\prime}(x ; \eta)$, which may depend on the parameter $\eta$.

48) The $c d f$ of MSBS is given by

$$
F(x ; \alpha, \beta, \gamma)=\int_{0}^{x} \frac{t^{-3 / 2}(t+\beta) \gamma}{\alpha \beta^{1 / 2}} \int_{0}^{\infty} u^{\gamma} e^{-2 u^{\gamma}} \phi\left(u a_{t}(\alpha, \beta)\right) d u,
$$


$x \geq 0$, where $\alpha, \beta, \gamma$ are positive parameters, $\phi(\cdot)$ is $p d f$ of standard normal distribution and $a_{x}(\alpha, \beta)=\left(\sqrt{\frac{x}{\beta}}-\sqrt{\frac{\beta}{x}}\right) / \alpha$. 49) The $c d f$ of IPL is given by

$$
F(x ; \alpha, \beta)=\left(1+\frac{\beta x^{-\alpha}}{1+\beta}\right) e^{-\beta x^{-\alpha}}, x \geq 0
$$

where $\alpha, \beta$ are positive parameters.

Remark 31. The IPL distribution of Barco et al. (2017) is the same as the Generalized Inverse Lindley (GIL) distribution of Asgharzadeh et al. (2016). The GIL distribution has been characterized in Hamedani (2017a).

50) The $c d f$ of WW is given by

$$
F(x ; \alpha, \beta, \lambda, \gamma)=1-\exp \left\{-\alpha\left[e^{\lambda x^{\gamma}}-1\right]^{\beta}\right\}, x \geq 0,
$$

where $\alpha, \beta, \lambda, \gamma$ are positive parameters.

Remark 32. The $c d f F(x ; \alpha, \beta, \lambda, a)=\left[1-\exp \left\{-\alpha\left[e^{\lambda x^{2}}-1\right]^{\beta}\right\}\right]^{a}, x \geq 0$ is of the form (50), which has been characterized in Hamedani (2017a). Similarly, $\operatorname{cdfs} F(x ; \theta, \gamma, a, b)=1-\left\{1-\left[1-\exp \left\{\left[-\frac{\theta}{\gamma}\left(e^{\gamma x}-1\right)\right]\right\}\right]^{a}\right\}^{b}, x \geq 0$ and $F(x ; \alpha, \beta, a, b)=1-\exp \left\{-a\left[\exp \left(e^{\alpha x-\beta / x}\right)-1\right]^{b}\right\}, x \geq 0$, which have been characterized in the Research Monograph by Hamedani and Maadooliat (2017).

51) The $c d f$ of BGKw-G is given by

$$
F(x ; a, b, m, n)=\frac{1}{B(m, n)} \int_{0}^{1-\left[1-G(x)^{a}\right]^{b}} t^{m-1}(1-t)^{n-1} d t
$$

$x \geq 0$, where $a, b, m, n$ are positive parameters.

Remark 33. The $c d f F(x ; \alpha, \beta)=\frac{1}{B(\alpha, \beta)} \int_{0}^{Q(x)} t^{\alpha-1}(1-t)^{\beta-1} d t, x \in \mathbb{R}$, where $Q(x)$ is an absolutely continuous $c d f$ has been introduced before and has been characterized in Hamedani (2016). Letting $Q(x)=1-\left[1-G(x)^{a}\right]^{b}$ in $F(x ; \alpha, \beta)$, we arrive at (101).

52) The $c d f$ of NLM is given by

$$
F(x ; \alpha)=e^{x^{\alpha} \ln 2}-1,0 \leq x \leq 1,
$$

where $\alpha$ is a positive parameter.

53) The $c d f$ of MOEBXII is given by

$$
F(x ; \alpha, \lambda, c, p)=\frac{1-\left\{1-\left[1-\left(1+x^{c}\right)^{-k}\right]^{\lambda}\right\}}{1-\bar{\alpha}\left\{1-\left[1-\left(1+x^{c}\right)^{-k}\right]^{\lambda}\right\}}, \quad x \geq 0
$$

where $\alpha, \lambda, c, p$ are all positive parameters and $\bar{\alpha}=1-\alpha$.

Remark 34. The $c d f F(x ; \alpha, \lambda, p)=\left\{\frac{1-(\bar{G}(x))^{\lambda}}{1-p(\bar{G}(x))^{\lambda}}\right\}^{\alpha}, x \in \mathbb{R}$, where $G(x)$ a baseline $c d f$ was introduced by Dias et al. (2016). Letting $\alpha=1$, renaming $p$ as $1-\alpha$ (for $\alpha>0)$ and $G(x)=\left(1-\left\{1-\left[1-\left(1+x^{c}\right)^{-k}\right]^{\lambda}\right\}\right)^{1 / \lambda}, x \geq 0$, we arrive at (53). Thus, $c d f(53)$ is a special case of Dias et al. distribution.

54) The $c d f$ of BNH is given by

$$
F(x ; \alpha, \lambda, a, b)=\frac{1}{B(a, b)} \int_{0}^{1-\exp \left\{1-(1+\lambda x)^{\alpha}\right\}} t^{a-1}(1-t)^{b-1} d t
$$


$x \geq 0$, where $\alpha, \lambda, a, b$ are all positive parameters.

Remark 35. As pointed out in Remark 33, the $c d f F(x ; \alpha, \beta)=\frac{1}{B(\alpha, \beta)} \int_{0}^{Q(x)} t^{\alpha-1}(1-t)^{\beta-1} d t, \quad x \in \mathbb{R}$, where $Q(x)$ is an absolutely continuous $c d f$ has been introduced before and has been characterized in Hamedani (2016). Letting $Q(x)=1-\exp \left\{1-(1+\lambda x)^{\alpha}\right\}$ in $F(x ; \alpha, \beta)$, we arrive at (54).

55) The $c d f$ of NTPW is given by

$$
F(x ; \theta, \beta)=\left(1-\exp \left\{-\left[e^{x^{\beta}}-1\right]^{2}\right\}\right)^{\theta}, x \geq 0,
$$

where $\theta, \beta$ are all positive parameters.

Remark 36. The $c d f F(x ; \theta, \beta)$ give in (55) has been characterized in Hamedani (2017a) under the name BrX-W.

56) The $p d f$ of GHT is given by

$$
f(x ; \alpha, \beta, \theta, \eta)=C_{1} x^{\alpha-1}\left(1+C_{2} x^{2 \alpha}\right)^{-\frac{\eta}{2 \alpha}-\frac{1}{2}}, \quad x>0,
$$

where $\alpha, \beta, \theta, \eta$ are all positive parameters, $C_{1}=\frac{\sqrt{2} \alpha \beta^{\alpha / 2}}{\sqrt{\pi} \eta^{\alpha / 2} \theta^{\alpha}}$ and $C_{2}=\frac{\beta^{\alpha}}{2 \eta^{\alpha} \theta^{2 \alpha}}$.

57) The $c d f$ of GE-G is given by

$$
F(x ; \alpha, \beta, \tau)=\frac{\beta^{\alpha}}{\Gamma(\alpha)} \int_{0}^{\frac{G(x, \tau)}{G(x ; \tau)}} t^{\alpha-1} e^{-\beta t} d t, \quad x \in \mathbb{R},
$$

where $\alpha, \beta$ are all positive parameters and $G(x ; \tau)$ is the baseline $c d f$ with corresponding $p d f g(x ; \tau)$, depending on the parameter vector $\tau$.

58) The $c d f$ of MB-F is given by

$$
\begin{gathered}
F(x ; a, b, c, d)=\frac{b^{a} d^{c}}{\Gamma(a+c)} \int_{0}^{\infty} z^{a+c-1} e^{-d z} \operatorname{erf}(\sqrt{x z / 2}) \times \\
{ }_{1} F_{1}(a ; a+c ;-(b-d) z) d z,
\end{gathered}
$$

for $x \geq 0$, and

$$
f(x ; a, b, c, d)=C x^{-1 / 2}\left(\frac{x}{2}+d\right)_{2}^{\frac{1}{2}-a-c} F_{1}\left(a ; a+c-\frac{1}{2} ; a+c ; \frac{d-b}{\frac{x}{2}+d}\right)
$$

and

$$
f(x ; a, b, c, d)=C x^{-1 / 2}\left(\frac{x}{2}+b\right)^{\frac{1}{2}-a-c}{ }_{2} F_{1}\left(c ; a+c-\frac{1}{2} ; a+c ; \frac{b-d}{\frac{x}{2}+b}\right)
$$

for $x>0,\left|\frac{d-b}{\frac{1}{2}+d}\right|<1$ and $\left|\frac{b-d}{\frac{x}{2}+b}\right|<1$ respectively, where $a, b, c, d$ are all positive parameters, $C=\frac{b^{a} d^{c} \Gamma\left(a+c-\frac{1}{2}\right)}{\sqrt{2 \pi} \Gamma(a+c)}$.

We will take up here the more interesting case of $b=d$. Similar conclusions can be made for the above two cases as well.

The $c d f$ of MB-F, for the case of $b=d$, is given by

$$
F(x ; a, b, c)=\frac{2 b^{a+c}}{\Gamma(a+c)} \int_{0}^{\infty} z^{2 a+2 c-1} e^{-b z^{2}} \operatorname{erf}\left(\sqrt{\frac{x}{2}} z\right) d z
$$

where $C_{1}=\frac{b^{a+c} \Gamma\left(a+c-\frac{1}{2}\right)}{\sqrt{2 \pi} \Gamma(a+c)}$.

59) The $c d f$ of M-W is given by 


$$
F(x ; \theta, \eta)=\frac{2}{\sqrt{\pi}} \int_{0}^{\frac{x^{\eta}}{2 \theta^{2}}} u^{1 / 2} e^{-u} d u, \quad x \geq 0,
$$

where $\theta, \eta$ are positive parameters.

60) The $c d f$ of EWE is given by

$$
F(x ; \alpha, \beta, \lambda, a)=\left[1-\exp \left(-\alpha\left(e^{\lambda x}-1\right)^{\beta}\right)\right]^{a}, \quad x \geq 0,
$$

where $\alpha, \beta, \lambda, a$ are positive parameters.

Remark 37. The $c d f F(x ; \alpha, \beta, \lambda, a)$ give in (60) is a special case of the Exponentiated Weibull Rayleigh (EWR) distribution of Elgarhy. The EWR distribution has been characterized in Hamedani (2017a).

61) The $c d f$ of $\alpha \mathrm{LT}$ are given by

$$
F(x ; \alpha)=\left\{\begin{array}{ll}
1-\frac{\log [\alpha-(\alpha-1) G(x)]}{\log \alpha}, & \text { if } \alpha>0, \alpha \neq 1 \\
G(x) & \text { if } \alpha=1
\end{array}, \quad x \in \mathbb{R},\right.
$$

where $\alpha$ is a parameter and $G(x)$ is the baseline $c d f$ with $p d f g(x)$.

62) The $c d f$ of TLOLL is given by

$$
F(x ; a, b, \xi)=\left\{1-\left[1-\frac{G(x ; \xi)^{a}}{G(x ; \xi)^{a}+\bar{G}(x ; \xi)^{a}}\right]^{2}\right\}^{b}, \quad x \in \mathbb{R}
$$

where $a, b$ are positive parameters and $G(x ; \xi)$ is the baseline $c d f$ with $p d f(x ; \xi)$, which depends on the parameter vector $\xi$.

Remark 38. The $c d f F(x ; a, b, \xi)$ given above is similar to the New Family (NF) of distributions of Alizadeh et al. (2015). The $c d f$ of NF distribution is given by

$$
F(x ; a, b, \alpha, \xi)=1-\left\{1-\left[\frac{G(x ; \xi)^{a}}{G(x ; \xi)^{a}+\bar{G}(x ; \xi)^{a}}\right]^{\alpha}\right\}^{b}, \quad x \in \mathbb{R}
$$

The NF distribution has been characterized in the Research Monograph by Hamedani and Maadooliat(2017).

63) The $c d f$ of TW-M is given by

$$
F(x ; \theta, \mu)=\frac{2}{\sqrt{\pi}} \int_{0}^{\theta(x-\mu)^{2}} u^{1 / 2} e^{-u} d u, \quad x \geq \mu
$$

where $\theta>0$ and $\mu \in \mathbb{R}$ are parameters.

64) The $c d f$ of GQHR is given by

$$
F(x ; a, b, c, d)=\left[1-e^{-\left(a x+\frac{b}{2} x^{2}+\frac{c}{3} x^{3}\right)}\right]^{d}, \quad x \geq 0,
$$

where $a \geq 0, c \geq 0, d>0$ and $b \geq-2 \sqrt{a c}$ are parameters.

Remark 39. The $c d f \quad F(x ; a, b, c, d)$ given in (64) is a special case of the Exponentiated Generalized (EG) class of distributions of Cordeiro et al. (2013). The $c d f$ of EG distribution is given by

$$
F(x ; \alpha, \beta)=\left[1-\{1-G(x)\}^{\alpha}\right]^{\beta}, \quad x \in \mathbb{R},
$$

where $G(x)$ is the baseline distribution. Taking $\alpha=1, G(x)=1-e^{-\left(a x+\frac{b}{2} x^{2}+\frac{c}{3} x^{3}\right)}, x>0$, we arrive at (64) .The EG distribution has been characterized in Hamedani (2016). The $c d f$ given in (64) is also a special case of the Kumaraswamy 
Quadratic Hazard Rate (KQHR) distribution of Elbatal and Aryal (2013). The KQHR has been characterized in Hamedani (2017).

65) The $c d f$ of NFPW is given by

$$
F(x ; a, b, \beta, \lambda)=\left[1-e^{\left(-a x^{\beta}\right)}\right]^{b} \times\left\{1+\lambda-\lambda\left[1-e^{\left(-a x^{\beta}\right)}\right]^{b}\right\}, \quad x \geq 0,
$$

where $a, b, \beta$ and $\lambda(|\lambda| \leq 1)$ are parameters.

Remark 40. The $c d f$ given in (65) is a special case of the Kumaraswamy-Tansmuted Exponentiated Modified Weibull (KwTEMW) distributions of Al-babtain et al. (2015). The $c d f$ of KwTEMW distribution is given by

$$
F(x ; \alpha, \beta, \theta, \gamma, a, b, \lambda)=1-\left\{1-\left[1-e^{-\left(\theta x+\gamma x^{\beta}\right)}\right]^{a \alpha} \times\left[1+\lambda-\lambda\left(1-e^{-\left(\theta x+\gamma x^{\beta}\right)}\right)^{\alpha}\right]^{a}\right\}^{b},
$$

$x \geq 0$, where $\alpha, \beta, \theta, \gamma, a, b, \lambda(|\lambda| \leq 1)$ are parameters. Taking $a=b=1$ and $\theta=0$, we arrive at (65). The KwTEMW distribution has been characterized in Hamedani (2018).

66) The $c d f$ of OLBXII is given by

$$
F(x ; \alpha, \beta, \sigma, \theta)=1-\frac{1+\theta\left[1+\left(\frac{x}{\sigma}\right)^{\alpha}\right]^{\beta}}{1+\theta} \exp \left\{\theta\left(1-\left[1+\left(\frac{x}{\sigma}\right)^{\alpha}\right]^{\beta}\right)\right\},
$$

$x \geq 0$, where $\alpha, \beta, \sigma, \theta$ are positive parameters.

67) The $c d f$ of DP is given by

$$
F(x ; \lambda, \gamma, \beta, \theta)=\frac{1-\exp \left(\theta\left[1+\lambda x^{-\gamma}\right]^{-\beta}\right)}{1-e^{\theta}}, x \geq 0,
$$

where $\lambda, \gamma, \beta, \theta$ are all positive parameters.

68) The $c d f$ of BXIIMW is given by

$$
F(x ; c, k, \alpha, \beta, \lambda)=1-\left(1+x^{c}\right)^{-k} e^{-\alpha x^{\beta} e^{\lambda x}}, x \geq 0,
$$

where $c, k, \alpha, \beta$ all positive and $\lambda \geq 0$ are parameters.

69) The $c d f$ of BXP is given by

$$
F(x ; \alpha, \beta, \delta)=\left(1-\exp \left\{-\left[\left(\frac{x}{\beta}\right)^{\alpha}-1\right]^{2}\right\}\right)^{\delta}, \quad x \geq \beta,
$$

where $\alpha, \beta, \delta$ are positive parameters.

Remark 41. The $c d f$ given in (69) is a special case of the Exponentiated Weibull-Pareto (EW-P) distributions of Afify et al. (2016). The $c d f$ of EW-P distribution is given by

$$
F(x ; \alpha, \theta, a, b)=\left(1-\exp \left\{-\left[\left(\frac{x}{\theta}\right)^{a}-1\right]^{b}\right\}\right)^{\alpha}, \quad x \geq \theta,
$$

where $\alpha, \theta, a, b$ are positive parameters. Taking $b=2$, we arrive at (69). The EW-P distribution has been characterized in Hamedani and Maadooliat (2017).

70) The $c d f$ of GCFW is given by

$$
F(x ; \alpha, \beta, \mu)=1-\exp \left\{-\exp \left\{\mu+\alpha \log H_{1}(x)+\beta \log H_{2}(x)\right\}\right\},
$$

where $\alpha>0, \beta>0$ and $\mu$ are parameters, $H_{i}(x), i=1,2$ are non-negative and nondecreasing for all $x \geq 0$ with $H_{i}(0)=0$, $\lim _{x \rightarrow \infty} H_{i}(x)=\infty$ and $h_{i}(x)=\frac{d}{d x} H_{i}(x)$. 
71) The $c d f$ of PLP is given by

$$
F(x ; \alpha, \beta, \theta, \lambda)=\frac{e^{\theta}-e^{-\theta\left[1-\lambda^{\alpha}\left(\lambda+x^{\beta}\right)^{-\alpha}\right]}}{e^{\theta}-1}, \quad x \geq 0,
$$

where $\alpha, \beta, \theta$ and $\lambda$ are positive parameters.

Remark 42. The $c d f$ given in (71) is a special case of the Truncated Weibull-G (TW-G) distributions of Najarzadegan et al. (2017). The TW-G distribution of Najarzadegan et al. is not new either since this distribution was introduced by Gomes et al. (2015), which is characterized in Hamedani and Maadooliat (2017).

72) The $c d f$ of MWP is given by

$$
F(x ; \alpha, \beta, \gamma, \theta)=\frac{\exp \left\{\theta\left(1-e^{-\alpha x-\beta x^{\gamma}}\right)\right\}}{e^{\theta}-1}, x \geq 0,
$$

where $\alpha, \beta \geq 0, \alpha+\beta>0, \gamma, \theta>0$ are parameters.

73) The $c d f$ of EPLG is given by

$$
F(x ; \alpha, \beta, \lambda, \theta)=\frac{(1-\theta)\left[1-\left(1+\frac{\lambda x^{\beta}}{\lambda+1}\right) e^{-\lambda x^{\beta}}\right]^{\alpha}}{1-\theta\left[1-\left(1+\frac{\lambda x^{\beta}}{\lambda+1}\right) e^{-\lambda x^{\beta}}\right]^{\alpha}}, x \geq 0
$$

where $\alpha, \beta, \lambda, \theta>0$ are parameters.

74) The $c d f$ of GMWPS is given by

$$
F(x ; \alpha, \beta, \gamma, \lambda, \theta)=\frac{C\left(\theta\left(1-\exp \left\{-\alpha x^{\gamma} e^{\lambda x}\right\}\right)^{\beta}\right)}{C(\theta)}, x \geq 0,
$$

where $\alpha, \beta, \theta>0$ and $\lambda, \gamma \geq 0$ are parameters and $C(\theta)=\sum_{n=1}^{\infty} a_{n} \theta^{n}, a_{i}^{\prime} s$ are nonnegative real numbers.

Remark 43. The $c d f$ given in (146) is a special case of the general form of

$$
F(x)=\frac{C\left(\theta t(x)^{\alpha}\right)}{C(\theta)}, \quad x \geq 0
$$

mentioned in Tahmasebi and Jafari (2016) in which they set $t(x)=1-\exp \left\{-\frac{\beta}{\gamma}\left(e^{\gamma x}-1\right)\right\}$. Taking $t(x)=\left(1-\exp \left\{-\alpha x^{\gamma} e^{\lambda x}\right\}\right)^{\beta}$ in the above $F(x)$, yields (74). The distribution of Tahmasebi and Jafari (2016) is characterized in Hamedani and Safavimanesh (2017b).

75) The $c d f$ of AMWOLLG (WLOG, for $\delta=\eta=1$ ) is given by

$$
F(x ; \alpha, \beta, \gamma, \lambda)=1-\exp \left\{-(\alpha(-\log \bar{G}(x ; \Theta))) e^{\gamma(-\log \bar{G}(x ; \Theta))}+e^{\lambda(-\log \bar{G}(x ; \Theta))-\beta}-e^{-\beta}\right\}, \quad x \in \mathbb{R}
$$

where $\alpha, \beta>0$ and $\lambda, \gamma \geq 0$ are parameters.

76) The $c d f$ of EPLPS is given by

$$
F(x ; \alpha, \beta, \lambda, \theta)=1-\frac{C\left(\theta-\theta\left[1-\left(1+\frac{\lambda x^{\beta}}{\lambda+1}\right) e^{-\lambda x^{\beta}}\right]^{\alpha}\right)}{C(\theta)}, x \geq 0,
$$

where $\alpha, \beta, \lambda, \theta>0$ are parameters and $C(\theta)=\sum_{n=1}^{\infty} a_{n} \theta^{n}, a_{i}^{\prime} s$ are nonnegative real numbers.

77) The $c d f$ of $\mathrm{W}-\mathrm{R}$ is given by

$$
F(x ; \gamma, c)=1-\exp \left\{-\frac{1}{\gamma^{c}}\left[-\log \left(1-F_{R}(x)\right)\right]^{c}\right\}, x \in \mathbb{R},
$$


where $\gamma, c>0$ are parameters and $F_{R}(x), f_{R}(x)$ are $c d f$ and $p d f$ of the special distribution taken in Ghosh and Nadarajah (2018).

78) The $c d f$ of WL is given by

$$
F(x ; \lambda)=\frac{1}{1-e^{-2 \pi}}\left(1-e^{-\lambda x}-\frac{\lambda x}{\lambda+1} e^{-\lambda x}\right)+\frac{2 \pi \lambda}{\lambda+1}\left(1-e^{-\lambda x}\right) \times\left(\frac{e^{-2 \pi \lambda}}{\left(1-e^{-2 \pi \lambda}\right)^{2}}\right),
$$

$0 \leq x \leq 2 \pi$, where $\lambda>0$ is a parameter.

79) The $c d f$ of BW-G is given by

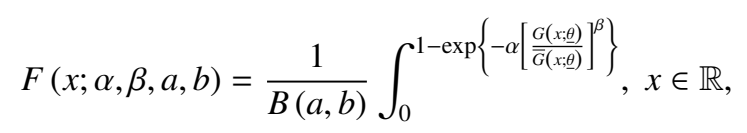

where $\alpha, \beta, a, b$ are positive parameters and $G(x ; \underline{\theta}), g(x ; \underline{\theta})$ are $c d f$ and $p d f$ of the baseline distribution which depends on the parameter vector $\underline{\theta}$.

Remark 44. The $c d f F(x ; \alpha, \beta)=\frac{1}{B(\alpha, \beta)} \int_{0}^{Q(x)} t^{\alpha-1}(1-t)^{\beta-1} d t, x \in \mathbb{R}$, where $Q(x)$ is an absolutely continuous $c d f$ has been introduced before and has been characterized in Hamedani (2016). Letting $Q(x)=1-\exp \left\{-\alpha\left[\frac{G(x ; \underline{\theta})}{\bar{G}(x ; \underline{\theta})}\right]^{\beta}\right\}$ in $F(x ; \alpha, \beta)$, we arrive at (79).

80) The $c d f$ of TWR is given by

$$
F(x \mid \beta, \lambda, \gamma(\mathbf{t}))=1-\exp \left[-\left(\frac{x}{\gamma(\mathbf{t})}\right)^{\beta}\right] \times\left\{1-\lambda+\lambda \exp \left[-\left(\frac{x}{\gamma(\mathbf{t})}\right)^{\beta}\right]\right\}, \quad x \geq 0,
$$

where $\beta>0, \lambda(|\lambda| \leq 1)$ are parameters and $\gamma(\mathbf{t})$ as a parameter depending on a covariate vector $\mathbf{t}=\left(1, t_{1}, \ldots, t_{p}\right)^{\prime}$ given by $\gamma(\mathbf{t})=\gamma_{0}+\gamma_{1} t_{1}+\ldots+\gamma_{p} t_{p}$.

\section{Characterizations of Distributions}

We present our characterizations (i) - (iv) in four subsections.

\subsection{Characterizations Based on Two Truncated Moments}

This subsection deals with the characterizations of distributions listed in Section 1 based on the ratio of two truncated moments. Our first characterization employs a theorem due to Glänzel (1987), see Theorem 1 of Appendix A . The result, however, holds also when the interval $H$ is not closed, since the condition of the Theorem is on the interior of $H$.

Proposition 1.1. Let $X: \Omega \rightarrow(0, \infty)$ be a continuous random variable and let $q_{2}(x)=\left(1-\left(1-\beta\left(a x+\frac{b}{2} x^{2}\right)\right)^{1 / \beta}\right)^{1-\alpha}$ and $q_{1}(x)=q_{2}(x)\left(1-\beta\left(a x+\frac{b}{2} x^{2}\right)\right)^{-1}$ for $x>0$. Then for $\beta<0$, the random variable $X$ has $c d f(1)$ if and only if the function $\xi$ defined in Theorem 1 is of the form

$$
\xi(x)=(1-\beta)\left(1-\beta\left(a x+\frac{b}{2} x^{2}\right)\right), \quad x>0 .
$$

Proof. Suppose the random variable $X$ has $c d f(1)$, then

$$
(1-F(x)) E\left[q_{1}(X) \mid X \geq x\right]=\frac{\alpha}{1-\beta}\left(1-\beta\left(a x+\frac{b}{2} x^{2}\right)\right)^{\frac{1}{\beta}-1}, \quad x>0,
$$

and

$$
(1-F(x)) E\left[q_{2}(X) \mid X \geq x\right]=\alpha\left(1-\beta\left(a x+\frac{b}{2} x^{2}\right)\right)^{1 / \beta}, \quad x>0 .
$$


Further,

$$
\xi(x) q_{1}(x)-q_{2}(x)=-\beta q_{2}(x)>0, \text { for } x>0
$$

Conversely, if $\xi$ is of the above form, then

$$
s^{\prime}(x)=\frac{\xi^{\prime}(x) q_{1}(x)}{\xi(x) q_{1}(x)-q_{2}(x)}=\frac{(1-\beta)(a+b x)}{1-\beta\left(a x+\frac{b}{2} x^{2}\right)}, \quad x>0,
$$

and consequently

$$
s(x)=-\frac{1-\beta}{\beta} \log \left\{1-\beta\left(a x+\frac{b}{2} x^{2}\right)\right\}, \quad x>0 .
$$

Now, according to Theorem $1, X$ has $c d f(1)$.

Corollary 1.1. Let $X: \Omega \rightarrow(0, \infty)$ be a continuous random variable and let $q_{2}(x)$ be as in Proposition 1.1 . For $\beta<0$, the random variable $X$ has $c d f(1)$ if and only if there exist functions $q_{1}$ and $\xi$ defined in Theorem 1 satisfying the following differential equation

$$
\frac{\xi^{\prime}(x) q_{1}(x)}{\xi(x) q_{1}(x)-q_{2}(x)}=\frac{(1-\beta)(a+b x)}{1-\beta\left(a x+\frac{b}{2} x^{2}\right)}, \quad x>0 .
$$

Remark 1.1. The general solution of the differential equation in Corollary 1.1 is

$$
\xi(x)=\left[1-\beta\left(a x+\frac{b}{2} x^{2}\right)\right]^{1-\frac{1}{\beta}}\left[\begin{array}{c}
-\int(1-\beta)(a+b x)\left[1-\beta\left(a x+\frac{b}{2} x^{2}\right)\right]^{\frac{1}{\beta}-2} \times \\
\left(q_{1}(x)\right)^{-1} q_{2}(x) d x+D
\end{array}\right]
$$

where $D$ is a constant. We like to point out that one set of functions satisfying the above differential equation is given in Proposition 1.1 with $D=0$. Clearly, there are other triplets $\left(q_{1}, q_{2}, \xi\right)$ which satisfy conditions of Theorem 1 .

Clearly, a Proposition, a Corollary and a Remark similar to the Proposition 1.1, Corollary 1.1 and Remark 1.1 can be stated for each of the distributions mentioned in the Introduction. For each of these distributions, we give below, the functions $q_{1}, q_{2}$ and $\xi$ corresponding to Theorem 1 .

2. $q_{1}(x)=\frac{\left\{1-p+p\left[1-e^{-\left(\frac{x}{\sigma}\right)^{\beta}}\right]^{\alpha}\left[1+\lambda e^{-\left(\frac{x}{\sigma}\right)^{\beta}}\right]^{\alpha}\right\}^{2}}{\left[1-\lambda+2 \lambda e^{-\left(\frac{x}{\sigma}\right)^{\beta}}\right]\left[1+\lambda e^{-\left(\frac{x}{\sigma}\right)^{\beta}}\right]^{\alpha-1}}, q_{2}(x)=q_{1}(x)\left[1-e^{-\left(\frac{x}{\sigma}\right)^{\beta}}\right]^{\alpha}$ and $\xi(x)=\frac{1}{2}\left\{1+\left[1-e^{-\left(\frac{x}{\sigma}\right)^{\beta}}\right]^{\alpha}\right\}$ for $x>0$.

6. $q_{1}(x)=\left[1-\exp \left\{-a(1+\eta x)^{-1 / \eta}\right\}\right]^{1-b}, q_{2}(x)=q_{1}(x) \exp \left\{-a(1+\eta x)^{-1 / \eta}\right\}$ and $\xi(x)=\frac{1}{2}\left\{1+\exp \left\{-a(1+\eta x)^{-1 / \eta}\right\}\right\}$ for $x>0$.

7. $q_{1}(x) \equiv 1, q_{2}(x)=\exp \left\{(G(x))^{\alpha}\right\}$ and $\xi(x)=\frac{1}{2}\left\{e+\exp \left\{(G(x))^{\alpha}\right\}\right\}$, for $x \in \mathbb{R}$.

10. $q_{1}(x)=\left\{1-p\left\{1-\left[1-(1+\lambda x)^{-\theta}\right]^{\alpha}\right\}\right\}^{2}, q_{2}(x)=q_{1}(x)\left[1-(1+\lambda x)^{-\theta}\right]^{\alpha}$ and $\xi(x)=\frac{1}{2}\left\{1+\left[1-(1+\lambda x)^{-\theta}\right]^{\alpha}\right\}$ for $x>0$.

12. $q_{1}(x)=\frac{\left[1-e^{-\left(\frac{\gamma}{x}\right)^{\beta}}\right]^{b+1} \exp \left\{\left[\frac{e^{-\left(\frac{\gamma}{x}\right)^{\beta}}}{1-e^{-\left(\frac{\gamma}{x}\right)^{\beta}}}\right]^{b}\right\}}{\left\{1-\lambda+2 \lambda \exp \left\{-\left[\frac{e^{-\left(\frac{\gamma}{x}\right)^{\beta}}}{1-e^{-\left(\frac{\gamma}{x}\right)^{\beta}}}\right]^{b}\right\}\right\}}, q_{2}(x)=q_{1}(x) e^{-b\left(\frac{\gamma}{x}\right)^{\beta}}$ and $\xi(x)=\frac{1}{2}\left\{1+e^{-b\left(\frac{\gamma}{x}\right)^{\beta}}\right\}$ for $x>0$.

14. $q_{1}(x) \equiv 1, q_{2}(x)=\exp \left\{-a\left(\log \left[1+\left(\frac{x}{s}\right)^{u}\right]\right)^{\beta}\right\}$ and $\xi(x)=\frac{1}{2} \exp \left\{-a\left(\log \left[1+\left(\frac{x}{s}\right)^{u}\right]\right)^{\beta}\right\}$ for $x>0$.

18. $q_{1}(x)=\frac{\left[1-\left(1-e^{-(\lambda x)^{2}}\right)^{\theta}\right]^{\beta+1}}{\left(1-e^{-(\lambda x)^{2}}\right)^{\theta \beta-1}} \exp \left\{-\alpha \frac{\left(1-e^{-(\lambda x)^{2}}\right)^{\theta \beta}}{\left[1-\left(1-e^{-(\lambda x)^{2}}\right)^{\theta}\right]^{\beta}}\right\}, q_{2}(x)=q_{1}(x) e^{-(\lambda x)^{2}}$ and $\xi(x)=\frac{1}{2} e^{-(\lambda x)^{2}}$ for $x>0$. 
19. $q_{1}(x)=\left\{1-\left[1-\left(\frac{\theta}{x}\right)^{\alpha}\right]^{a}\left[1+\lambda\left(\frac{\theta}{x}\right)^{\alpha}\right]^{a}\right\}^{1-b}, q_{2}(x)=q_{1}(x)\left\{\left[1-\left(\frac{\theta}{x}\right)^{\alpha}\right]\left[1+\lambda\left(\frac{\theta}{x}\right)^{\alpha}\right]\right\}^{a}$ and $\xi(x)=\frac{1}{2}\left\{1+\left[1-\left(\frac{\theta}{x}\right)^{\alpha}\right]^{a}\left[1+\lambda\left(\frac{\theta}{x}\right)^{\alpha}\right]^{a}\right\}$ for $x>0$.

21. $q_{1}(x)=x^{1-\alpha}\left[\delta-(\delta-1)\left(\frac{x}{x+\beta}\right)^{\alpha}\right]^{2}, q_{2}(x)=q_{1}(x)(x+\beta)^{-1}$ and $\xi(x)=\frac{\alpha}{\alpha+1}(x+\beta)^{-1}$ for $x>0$.

23. $q_{1}(x)=(1+x)^{-1}, q_{2}(x)=q_{1}(x) e^{-\theta(x-\eta)}$ and $\xi(x)=\frac{1}{2}\left(1+e^{-\theta(x-\eta)}\right)$ for $0<x<\eta$.

24. $q_{1}(x)=\left[1-\lambda+2 \lambda\left(1-e^{-\left(\frac{\theta}{x}\right)^{\beta}}\right)^{\alpha}\right]^{-1}, q_{2}(x)=q_{1}(x)\left(1-e^{-\left(\frac{\theta}{x}\right)^{\beta}}\right)$ and $\xi(x)=\frac{\alpha}{\alpha+1}\left(1-e^{-\left(\frac{\theta}{x}\right)^{\beta}}\right)$ for $x>0$.

28. $q_{1}(x)=e^{-\lambda(\beta x)^{\alpha}}\left[1-e^{-\lambda}-\pi+\pi \exp \left(-\lambda+\lambda e^{-(\beta x)^{\alpha}}\right)\right]^{2}, q_{2}(x)=q_{1}(x) e^{-(\beta x)^{\alpha}}$ and $\xi(x)=\frac{1}{2} e^{-(\beta x)^{\alpha}}$ for $x>0$.

29. $q_{1}(x)=\left[\sin \left(\frac{\pi}{2} e^{-\theta x}\right)\right]^{-1}, q_{2}(x)=q_{1}(x) e^{-\theta x}$ and $\xi(x)=\frac{1}{2} e^{-\theta x}$ for $x>0$.

30. $q_{1}(x)=\left[1-\exp \left(\lambda\left(\left(1+x^{\alpha}\right)^{-\beta}-1\right)\right)\right]^{1-a}, q_{2}(x)=q_{1}(x) \exp \left(\lambda\left(\left(1+x^{\alpha}\right)^{-\beta}-1\right)\right)$ and $\xi(x)=\frac{1}{2}\left\{\exp \left(\lambda\left(\left(1+x^{\alpha}\right)^{-\beta}-1\right)\right)+1\right\}$ for $x>0$.

38. $q_{1}(x)=\left[1-\left(\frac{x}{\lambda}\right)^{a \theta}\right]^{1-b}, q_{2}(x)=q_{1}(x)\left(\frac{x}{\lambda}\right)^{a \theta}$ and $\xi(x)=\frac{1}{2}\left[1+\left(\frac{x}{\lambda}\right)^{a \theta}\right]$ for $0<x<\lambda$.

42. $q_{1}(x)=(\lambda x)^{-\omega}[(1-\beta) \Gamma(\omega+1)+2 \beta \Gamma(\omega+1, \lambda x)]^{-1}, q_{2}(x)=x q_{1}(x)$ and $\xi(x)=x+\frac{1}{\lambda}$ for $x>0$.

43. $q_{1}(x)=\left[1-\lambda+2 \lambda\left(\frac{x_{0}}{x}\right)^{\alpha}\right]^{-1}\left[1+\lambda\left(\frac{x_{0}}{x}\right)^{\alpha}\right]^{1-a}\left\{\left(\frac{x_{0}}{x}\right)^{\alpha}\left[1-\lambda+\lambda\left(\frac{x_{0}}{x}\right)^{\alpha}\right]\right\}^{1-b}, q_{2}(x)=q_{1}(x)\left[1-\left(\frac{x_{0}}{x}\right)^{\alpha}\right]^{a}$ and $\xi(x)=$ $\frac{1}{2}\left\{1+\left[1-\left(\frac{x_{0}}{x}\right)^{\alpha}\right]^{a}\right\}$ for $x>x_{0}$.

44. $q_{1}(x)=\left(1+(-\log (\bar{G}(x ; \eta)))^{c}\right)^{k-1}, q_{2}(x)=q_{1}(x)\left(1+(-\log (\bar{G}(x ; \eta)))^{c}\right)^{-1}$ and $\xi(x)=\frac{1}{2}\left(1+(-\log (\bar{G}(x ; \eta)))^{c}\right)^{-1}$ for $x \in \mathbb{R}$.

47. $q_{1}(x)=\left[1-(1-\delta)\left(1+x^{c}\right)^{-1} e^{-\alpha H(x ; \eta)}\right]^{2}, q_{2}(x)=q_{1}(x)\left(1+x^{c}\right)^{-1} e^{-\alpha H(x ; \eta)}$ and $\xi(x)=\frac{1}{2}\left(1+x^{c}\right)^{-1} e^{-\alpha H(x ; \eta)}$ for $x>0$.

48. $q_{1}(x)=\left\{(x+\beta) \int_{0}^{\infty} u^{\gamma} e^{-2 u^{\gamma}} \phi\left(u a_{x}(\alpha, \beta)\right) d u\right\}^{-1}, q_{2}(x)=x^{-1} q_{1}(x)$ and $\xi(x)=\frac{1}{2} x^{-1 / 2}$ for $x>0$.

50. $q_{1}(x) \equiv 1, q_{2}(x)=\exp \left\{-\alpha\left[e^{\lambda x^{\gamma}}-1\right]^{\beta}+\lambda x^{\gamma}\right\}$ and $\xi(x)=\frac{1}{2} \exp \left\{-\alpha\left[e^{\lambda x^{\gamma}}-1\right]^{\beta}\right\}$ for $x>0$.

52. $q_{1}(x)=e^{-x^{\alpha} \ln 2}, q_{2}(x)=q_{1}(x)(\ln 2) x^{\alpha}$ and $\xi(x)=\frac{1}{2}\left\{1+(\ln 2) x^{\alpha}\right\}$ for $0<x<1$.

55. $q_{1}(x)=x^{\alpha}\left(1=C_{2} x^{2 \alpha}\right)^{-1 / 2}, q_{2}(x)=q_{1}(x)\left(1=C_{2} x^{2 \alpha}\right)^{-1 / 2}$ and $\xi(x)=\frac{\eta}{\eta+\alpha}\left(1+C_{2} x^{2 \alpha}\right)^{-1 / 2}$ for $x>0$.

56. $q_{1}(x)=\bar{G}(x ; \tau)^{\alpha+1} \exp \left\{\frac{\beta G(x ; \tau)}{\bar{G}(x ; \tau)}\right\}, q_{2}(x)=q_{1}(x) G(x ; \tau)^{\alpha}$ and $\xi(x)=\frac{1}{2}\left(1+G(x ; \tau)^{\alpha}\right)$ for $x \in \mathbb{R}$.

57. $q_{1}(x)=x^{1 / 2}, q_{2}(x)=q_{1}(x)\left(\frac{x}{2}+b\right)^{\varepsilon}$ and $\xi(x)=\frac{2(a+c)-3}{2(a+c)-(3+2 \varepsilon)}\left(\frac{x}{2}+b\right)^{\varepsilon}$ for $x>0$ and $\varepsilon$ any positive number.

58. $q_{1}(x)=x^{-\eta / 2}, q_{2}(x)=q_{1}(x) e^{-\frac{x^{\eta}}{2 \theta^{2}}}$ and $\xi(x)=\frac{1}{2} e^{-\frac{x^{\eta}}{2 \theta^{2}}}$ for $x>0$.

60. $q_{1}(x)=[\alpha-(\alpha-1) G(x)], q_{2}(x)=q_{1}(x) G(x)$ and $\xi(x)=\frac{1}{2}\{1+G(x)\}$ for $x \in \mathbb{R}$.

62. $q_{1}(x)=(x-\mu)^{-1}, q_{2}(x)=q_{1}(x) e^{-\theta(x-\mu)^{2}}$ and $\xi(x)=\frac{1}{2} e^{-\theta(x-\mu)^{2}}$ for $x>\mu$.

65. $q_{1}(x) \equiv 1, q_{2}(x)=\exp \left\{\theta\left(1-\left[1+\left(\frac{x}{\sigma}\right)^{\alpha}\right]^{\beta}\right)\right\}$ and $\xi(x)=\frac{1}{2} \exp \left\{\theta\left(1-\left[1+\left(\frac{x}{\sigma}\right)^{\alpha}\right]^{\beta}\right)\right\}$ for $x>0$.

66. $q_{1}(x)=\exp \left(-\theta\left[1+\lambda x^{-\gamma}\right]^{-\beta}\right), q_{2}(x)=q_{1}(x)\left[1+\lambda x^{-\gamma}\right]^{-\beta}$ and $\xi(x)=\frac{1}{2}\left\{1+\left(1+\lambda x^{-\gamma}\right)^{-\beta}\right\}$ for $x>0$.

67. $q_{1}(x) \equiv 1, q_{2}(x)=\left(1+x^{c}\right)^{-k} e^{-\alpha x^{\beta} e^{\lambda x}}$ and $\xi(x)=\frac{1}{2}\left(1+x^{c}\right)^{-k} e^{-\alpha x^{\beta} e^{\lambda x}}$ for $x>0$.

69. $q_{1}(x) \equiv 1, q_{2}(x)=\exp \left\{-\exp \left\{\mu+\alpha \log H_{1}(x)+\beta \log H_{2}(x)\right\}\right\}$ and $\xi(x)=\frac{1}{2} \exp \left\{-\exp \left\{\mu+\alpha \log H_{1}(x)+\beta \log H_{2}(x)\right\}\right\}$ for $x>0$.

71. $q_{1}(x)=\exp \left\{-\theta\left(1-e^{-\alpha x-\beta x^{\gamma}}\right)\right\}, q_{2}(x)=q_{1}(x) e^{-\alpha x-\beta x^{\gamma}}$ and $\xi(x)=\frac{1}{2} e^{-\alpha x-\beta x^{\gamma}}$ for $x>0$.

72. $q_{1}(x)=\left(1-\theta\left[1-\left(1+\frac{\lambda x^{\beta}}{\lambda+1}\right) e^{-\lambda x^{\beta}}\right]^{\alpha}\right)^{2}, q_{2}(x)=q_{1}(x)\left[1-\left(1+\frac{\lambda x^{\beta}}{\lambda+1}\right) e^{-\lambda x^{\beta}}\right]^{\alpha}$ and $\xi(x)=\frac{1}{2}\left\{1+\left[1-\left(1+\frac{\lambda x^{\beta}}{\lambda+1}\right) e^{-\lambda x^{\beta}}\right]^{\alpha}\right\}$ for $x>0$. 
74. $q_{1}(x)=\left(\frac{1}{\bar{G}(x ; \Theta)}\left\{\alpha e^{\gamma(-\log \bar{G}(x ; \Theta))}(\gamma(-\log \bar{G}(x ; \Theta))+1)+\lambda e^{\lambda(-\log \bar{G}(x ; \Theta))-\beta}\right\}\right.$ $\left.\times \exp \left\{-(\alpha(-\log \bar{G}(x ; \Theta))) e^{\gamma(-\log \bar{G}(x ; \Theta))}+e^{\lambda(-\log \bar{G}(x ; \Theta))-\beta}-e^{-\beta}\right\}\right)^{-1}, q_{2}(x)=q_{1}(x) G(x ; \Theta)$ and $\xi(x)=\frac{1}{2}\{1+G(x ; \Theta)\}$ for $x>0$.

75. $q_{1}(x)=\left(C^{\prime}\left(\theta-\theta\left[1-\left(1+\frac{\lambda x^{\beta}}{\lambda+1}\right) e^{-\lambda x^{\beta}}\right]^{\alpha}\right)\right)^{-1}, q_{2}(x)=q_{1}(x)\left[1-\left(1+\frac{\lambda x^{\beta}}{\lambda+1}\right) e^{-\lambda x^{\beta}}\right]^{\alpha}$ and $\xi(x)=\frac{1}{2}\left\{1+\left[1-\left(1+\frac{\lambda x^{\beta}}{\lambda+1}\right) e^{-\lambda x^{\beta}}\right]^{\alpha}\right\}$ for $x>0$.

76. $q_{1}(x) \equiv 1, q_{2}(x)=\exp \left\{-\frac{1}{\gamma^{c}}\left[-\log \left(1-F_{R}(x)\right)\right]^{c}\right\}$ and $\xi(x)=\frac{1}{2} \exp \left\{-\frac{1}{\gamma^{c}}\left[-\log \left(1-F_{R}(x)\right)\right]^{c}\right\}$ for $x \in \mathbb{R}$.

77. $q_{1}(x)=\left[\frac{1+x}{1-e^{-2 \pi \lambda}}+\frac{2 \pi e^{-2 \pi \lambda}}{\left(1-e^{-2 \pi \lambda}\right)^{2}}\right]^{-1}, q_{2}(x)=q_{1}(x) e^{-\lambda x}$ and $\xi(x)=\frac{1}{2}\left[e^{-\lambda x}+e^{-2 \pi \lambda}\right]$ for $x \in(0,2 \pi)$.

78. $q_{1}(x)=\left\{1-\lambda+2 \lambda \exp \left[-\left(\frac{x}{\gamma(\mathbf{t})}\right)^{\beta}\right]\right\}^{-1}, q_{2}(x)=q_{1}(x) \exp \left[-\left(\frac{x}{\gamma(\mathbf{t})}\right)^{\beta}\right]$ and $\xi(x)=\frac{1}{2} \exp \left[-\left(\frac{x}{\gamma(\mathbf{t})}\right)^{\beta}\right]$ for $x>0$.

\subsection{Characterization in Terms of Hazard Function}

The hazard function, $h_{F}$, of a twice differentiable distribution function, $F$, satisfies the following first order differential equation $\frac{f^{\prime}(x)}{f(x)}=\frac{h_{F}^{\prime}(x)}{h_{F}(x)}-h_{F}(x)$. It should be mentioned that for many univariate continuous distributions, the above equation is the only differential equation available in terms of the hazard function. In this subsection we present non-trivial characterizations of EL (for $k=1$ ), ETWG (for $\lambda=0$ ), KumGEV, GEG, ELG (for $\alpha=1$ ), TWFr (for $\lambda=0$ ), GWBXII, WBX, KwTP, MOEIP, UTL, TEF (for $\lambda=0$ ), GBXIIP (for $a=1$ ), TTL (for $\lambda=0$ ), Kw-PF, GBG, WW, NLM, $\alpha$ TL , OLBXII, BXIIMW, GCFW, MWP, AMWOLLG, EPLPS, W-G and TWR distributions in terms of the hazard function, which are not of the above trivial form.

Proposition 2.1. Let $X: \Omega \rightarrow(0, \infty)$ be a continuous random variable. The random variable $X$ has $c d f(1)$ (for $k=1)$ if and only if its hazard function $h_{F}(x)$ satisfies the following differential equation

$$
h_{F}^{\prime}(x)-\frac{\theta^{2}}{\theta^{2}(x+1)+(1+\theta)} h_{F}(x)=-\frac{\theta^{3}(1+\theta)\left[\theta^{2}(x+1)+(1+\theta)\right]}{\left[\theta^{2}(1+\theta) x+(1+\theta)^{3}\right]^{2}}, \quad x>0 .
$$

Proof. If $X$ has $c d f(1)$ for $k=1$, then clearly the above differential equation holds. If the differential equation holds, then

$$
\begin{aligned}
\frac{d}{d x}\left\{\left[\theta^{2}(x+1)+(1+\theta)\right]^{-1} h_{F}(x)\right\} & =-\frac{\theta^{3}(1+\theta)}{\left[\theta^{2}(1+\theta) x+(1+\theta)^{3}\right]^{2}} \\
& =\theta \frac{d}{d x}\left\{\left[\theta^{2}(1+\theta) x+(1+\theta)^{3}\right]^{-1}\right\}
\end{aligned}
$$

from which we arrive at the hazard function corresponding to the $c d f(1)$.

A Proposition similar to that of Proposition 2.1 will be stated (without proof) for each one of the distributions listed in subsection 2.1.

Proposition 2.2. Let $X: \Omega \rightarrow(0, \infty)$ be a continuous random variable. The random variable $X$ has $c d f(2)$ (for $\lambda=0)$, if and only if its hazard function $h_{F}(x)$ satisfies the following differential equation

$$
h_{F}^{\prime}(x)+\frac{\beta x^{\beta-1}}{\sigma^{\beta}} h_{F}(x)=\frac{\alpha \beta}{\sigma} e^{-\left(\frac{x}{\sigma}\right)^{\beta}} \frac{d}{d x}\left\{\frac{\left(\frac{x}{\sigma}\right)^{\beta-1}\left[1-e^{-\left(\frac{x}{\sigma}\right)^{\beta}}\right]^{\alpha-1}}{\left[1-e^{-\left(\frac{x}{\sigma}\right)^{\beta}}\right]\left\{1-p+p\left[1-e^{-\left(\frac{x}{\sigma}\right) \beta}\right]^{\alpha}\left[1+\lambda e^{-\left(\frac{x}{\sigma}\right) \beta}\right]^{\alpha}\right\}}\right\}, x>0 .
$$

Proposition 2.3. Let $X: \Omega \rightarrow(0, \infty)$ be a continuous random variable. The random variable $X$ has $c d f(6)$, if and only if its hazard function $h_{F}(x)$ satisfies the following differential equation

$$
h_{F}^{\prime}(x)-a(1+\eta x)^{-\left(\frac{1}{\eta}+1\right)} h_{F}(x)=a b \exp \left\{-a(1+\eta x)^{-1 / \eta}\right\} \frac{d}{d x}\left\{\frac{(1+\eta x)^{-\left(\frac{1}{\eta}+1\right)}}{1-\exp \left\{-a(1+\eta x)^{-1 / \eta}\right\}}\right\}, x>0 .
$$


Proposition 2.4. Let $X: \Omega \rightarrow \mathbb{R}$ be a continuous random variable. The random variable $X$ has $c d f(7)$, if and only if its hazard function $h_{F}(x)$ satisfies the following differential equation

$$
h_{F}^{\prime}(x)-\alpha g(x)(G(x))^{\alpha-1} h_{F}(x)=\alpha \exp \left\{(G(x))^{\alpha}\right\} \frac{d}{d x}\left\{\frac{g(x)(G(x))^{\alpha-1}}{e-\exp \left\{(G(x))^{\alpha}\right\}}\right\}, x \in \mathbb{R} .
$$

Proposition 2.5. Let $X: \Omega \rightarrow(0, \infty)$ be a continuous random variable. The random variable $X$ has $c d f(10)$, for $\alpha=1$, if and only if its hazard function $h_{F}(x)$ satisfies the following differential equation

$$
h_{F}^{\prime}(x)+\lambda(1+\lambda x)^{-1} h_{F}(x)=-\frac{p \lambda^{2} \theta^{2}(1+\lambda x)^{-(\theta+2)}}{\left[1-p(1+\lambda x)^{-\theta}\right]^{2}}, \quad x>0 .
$$

Proposition 2.6. Let $X: \Omega \rightarrow(0, \infty)$ be a continuous random variable. The random variable $X$ has $c d f(12)$, for $\lambda=0$, if and only if its hazard function $h_{F}(x)$ satisfies the following differential equation

$$
h_{F}^{\prime}(x)-b \beta x^{-(\beta+1)} h_{F}(x)=b \beta \gamma^{\beta} e^{-b\left(\frac{\gamma}{x}\right)^{\beta}} \frac{d}{d x}\left\{\frac{x^{-(\beta+1)}}{\left[1-e^{-\left(\frac{\gamma}{x}\right)^{\beta}}\right]^{b+1}}\right\}, \quad x>0 .
$$

Proposition 2.7. Let $X: \Omega \rightarrow(0, \infty)$ be a continuous random variable. The random variable $X$ has $c d f(14)$, if and only if its hazard function $h_{F}(x)$ satisfies the following differential equation

$$
h_{F}^{\prime}(x)-(u-1) x^{-1} h_{F}(x)=\alpha \beta u x^{u-1} \frac{d}{d x}\left\{\frac{\left(\log \left[1+\left(\frac{x}{s}\right)^{u}\right]\right)^{\beta-1}}{x^{u}+s^{u}}\right\}, \quad x>0 .
$$

Proposition 2.8. Let $X: \Omega \rightarrow(0, \infty)$ be a continuous random variable. The random variable $X$ has $c d f(18)$, if and only if its hazard function $h_{F}(x)$ satisfies the following differential equation

$$
h_{F}^{\prime}(x)+2 \lambda^{2} x h_{F}(x)=2 \alpha \beta \lambda^{2} \theta e^{-(\lambda x)^{2}} \frac{d}{d x}\left\{\frac{x\left(1-e^{-(\lambda x)^{2}}\right)^{\theta \beta-1}}{\left[1-\left(1-e^{-(\lambda x)^{2}}\right)^{\theta}\right]^{\beta+1}}\right\}, \quad x>0 .
$$

Proposition 2.9. Let $X: \Omega \rightarrow(0, \infty)$ be a continuous random variable. The random variable $X$ has $p d f(38)$, if and only if its hazard function $h_{F}(x)$ satisfies the following differential equation

$$
\begin{gathered}
h_{F}^{\prime}(x)+\frac{\alpha+1}{x} h_{F}(x)= \\
a b \alpha \theta^{\alpha} x^{-(\alpha+1)} \frac{d}{d x}\left\{\frac{\left[1-\lambda+2 \lambda\left(\frac{\theta}{x}\right)^{\alpha}\right]\left\{\left[1-\left(\frac{\theta}{x}\right)^{\alpha}\right]\left[1+\lambda\left(\frac{\theta}{x}\right)^{\alpha}\right]\right\}^{a-1}}{1-\left[1-\left(\frac{\theta}{x}\right)^{\alpha}\right]^{a}\left[1+\lambda\left(\frac{\theta}{x}\right)^{\alpha}\right]^{a}}\right\}, \quad x>0 .
\end{gathered}
$$

Proposition 2.10. Let $X: \Omega \rightarrow(0, \infty)$ be a continuous random variable. The random variable $X$ has $c d f(21)$, if and only if its hazard function $h_{F}(x)$ satisfies the following differential equation

$$
h_{F}^{\prime}(x)-\frac{\alpha-1}{x+\beta} h_{F}(x)=\alpha \beta(x+\beta)^{\alpha-1} \frac{d}{d x}\left\{\frac{x^{\alpha-1}}{\left[(x+\beta)^{\alpha}-x^{\alpha}\right]\left[\delta(x+\beta)^{\alpha}-(\delta-1) x^{\alpha}\right]}\right\}, x>0 .
$$

Proposition 2.11. Let $X: \Omega \rightarrow(0, \eta)$ be a continuous random variable. The random variable $X$ has $p d f(46)$, if and only if its hazard function $h_{F}(x)$ satisfies the following differential equation

$$
h_{F}^{\prime}(x)+\theta h_{F}(x)=\frac{\theta^{2}}{(1+\theta)^{2}}\left\{\frac{1+\theta+\theta\left[x+\theta(1+x)^{2}\right] e^{-\theta x}-(1+\theta+\theta \eta) e^{-\theta \eta}}{\left[(1+\theta+\theta x) e^{-\theta x}-(1+\theta+\theta \eta) e^{-\theta \eta}\right]^{2}}\right\}, 0<x<\eta .
$$


Proposition 2.12. Let $X: \Omega \rightarrow(0, \infty)$ be a continuous random variable. The random variable $X$ has $c d f(24)$, for $\lambda=0$, if and only if its hazard function $h_{F}(x)$ satisfies the following differential equation

$$
h_{F}^{\prime}(x)-\beta \theta^{\beta} x^{-(\beta+1)} h_{F}(x)=\alpha \beta \theta^{\beta} e^{-\left(\frac{\theta}{x}\right)^{\beta}}\left\{\frac{x^{-(\beta+2)}\left[\left(\beta+1-\beta \theta^{\beta} x\right) e^{-\left(\frac{\theta}{x}\right)^{\beta}}-(\beta+1)\right]}{\left(1-e^{-\left(\frac{\theta}{x}\right)^{\beta}}\right)^{2}}\right\}, x>0 .
$$

Proposition 2.13. Let $X: \Omega \rightarrow(0, \infty)$ be a continuous random variable. The random variable $X$ has $c d f(30)$, for $a=1$, if and only if its hazard function $h_{F}(x)$ satisfies the following differential equation

$$
h_{F}^{\prime}(x)-\frac{\alpha-1}{x} h_{F}(x)=-a \alpha^{2} \beta \lambda x^{2(\alpha-1)}\left(1+x^{\alpha}\right)^{-\beta-2}+a \alpha \beta \lambda e^{-\lambda} x^{\alpha-1} \frac{d}{d x}\left\{\frac{\left(1+x^{\alpha}\right)^{-\beta-1}}{\exp \left(\lambda\left(\left(1+x^{\alpha}\right)^{-\beta}-1\right)\right)-e^{-\lambda}}\right\}, x>0
$$

Proposition 2.14. Let $X: \Omega \rightarrow(0, \infty)$ be a continuous random variable. The random variable $X$ has $c d f(34)$, for $\lambda=0$, if and only if its hazard function $h_{F}(x)$ satisfies the following differential equation

$$
h_{F}^{\prime}(x)-\frac{\alpha}{1+\alpha x} h_{F}(x)=-\alpha \theta^{3}(1+\alpha x)(\theta+\alpha+\alpha \theta x)^{-2}, \quad x>0 .
$$

Proposition 2.15. Let $X: \Omega \rightarrow(0, \lambda)$ be a continuous random variable. The random variable $X$ has $c d f(38)$, if and only if its hazard function $h_{F}(x)$ satisfies the following differential equation

$$
h_{F}^{\prime}(x)-\frac{a \theta-1}{x} h_{F}(x)=\frac{a^{2} b \theta^{2}}{\lambda^{2}}\left(\frac{x}{\lambda}\right)^{2(a \theta-1)}\left[1-\left(\frac{x}{\lambda}\right)^{a \theta}\right]^{-2}, 0<x<\lambda .
$$

Proposition 2.16. Let $X: \Omega \rightarrow \mathbb{R}$ be a continuous random variable. The random variable $X$ has $c d f(44)$, if and only if its hazard function $h_{F}(x)$ satisfies the following differential equation

$$
h_{F}^{\prime}(x)-\frac{g^{\prime}(x ; \eta)}{g(x ; \eta)} h_{F}(x)=\operatorname{ckg}(x ; \eta) \frac{d}{d x}\left\{\frac{(-\log (\bar{G}(x ; \eta)))^{c-1}\left(1+(-\log (\bar{G}(x ; \eta)))^{c}\right)}{\bar{G}(x ; \eta)}\right\}, \quad x \in \mathbb{R} .
$$

Proposition 2.17. Let $X: \Omega \rightarrow(0, \infty)$ be a continuous random variable. The random variable $X$ has $c d f(50)$, if and only if its hazard function $h_{F}(x)$ satisfies the following differential equation

$$
h_{F}^{\prime}(x)-\frac{\gamma-1}{x} h_{F}(x)=\alpha \beta(\beta-1) \lambda^{2} \gamma^{2} x^{2(\gamma-1)} e^{\lambda x^{\gamma}}\left[e^{\lambda x^{\gamma}}-1\right]^{\beta-2}, x>0 .
$$

Proposition 2.18. Let $X: \Omega \rightarrow(0,1)$ be a continuous random variable. The random variable $X$ has $p d f(104)$, if and only if its hazard function $h_{F}(x)$ satisfies the following differential equation

$$
h_{F}^{\prime}(x)-\frac{\alpha-1}{x} h_{F}(x)=0,0<x<1 .
$$

Proposition 2.19. Let $X: \Omega \rightarrow \mathbb{R}$ be a continuous random variable. The random variable $X$ has $c d f(60)$, if and only if its hazard function $h_{F}(x)$ satisfies the following differential equation

$$
h_{F}^{\prime}(x)-\frac{g^{\prime}(x)}{g(x)} h_{F}(x)=(\alpha-1) g(x) \frac{d}{d x}\left\{\frac{[\alpha-(\alpha-1) G(x)]^{-1}}{\log [\alpha-(\alpha-1) G(x)]}\right\} x \in \mathbb{R} .
$$

Proposition 2.20. Let $X: \Omega \rightarrow(0, \infty)$ be a continuous random variable. The random variable $X$ has $c d f(65)$, if and only if its hazard function $h_{F}(x)$ satisfies the following differential equation

$$
h_{F}^{\prime}(x)-\frac{\alpha-1}{x} h_{F}(x)=\theta^{2} \alpha \beta \sigma^{-\alpha} x^{\alpha-1} \frac{d}{d x}\left\{\frac{\left[1+\left(\frac{x}{\sigma}\right)^{\alpha}\right]^{2 \beta-1}}{1+\theta\left[1+\left(\frac{x}{\sigma}\right)^{\alpha}\right]^{\beta}}\right\}, x>0 .
$$


Proposition 2.21. Let $X: \Omega \rightarrow(0, \infty)$ be a continuous random variable. The random variable $X$ has $c d f(67)$ if and only if its hazard function $h_{F}(x)$ satisfies the differential equation

$$
h_{F}^{\prime}(x)+\frac{c x^{c-1}}{1+x^{c}} h_{F}(x)=\left(1+x^{c}\right)^{-1} \frac{d}{d x}\left\{k c x^{c-1}+\left(1+x^{c}\right) \alpha e^{\lambda x} x^{\beta-1}(\beta+\lambda x)\right\}, x>0 .
$$

Proposition 2.22. Let $X: \Omega \rightarrow(0, \infty)$ be a continuous random variable. The random variable $X$ has $c d f(69)$ if and only if its hazard function $h_{F}(x)$ satisfies the differential equation

$$
h_{F}^{\prime}(x)-\left(\alpha \frac{h_{1}(x)}{H_{1}(x)}+\beta \frac{h_{2}(x)}{H_{2}(x)}\right) h_{F}(x)=\exp \left\{\mu+\alpha \log H_{1}(x)+\beta \log H_{2}(x)\right\} \frac{d}{d x}\left\{\left(\alpha \frac{h_{1}(x)}{H_{1}(x)}+\beta \frac{h_{2}(x)}{H_{2}(x)}\right)\right\}, x>0 .
$$

Proposition 2.23. Let $X: \Omega \rightarrow(0, \infty)$ be a continuous random variable. The random variable $X$ has $c d f(71)$ if and only if its hazard function $h_{F}(x)$ satisfies the differential equation

$$
h_{F}^{\prime}(x)+\left(\alpha+\beta \gamma x^{\gamma-1}\right) h_{F}(x)=\theta e^{-\alpha x-\beta x^{\gamma}} \frac{d}{d x}\left\{\frac{\alpha x+\beta \gamma x^{\gamma-1}}{\exp \left\{\theta e^{-\alpha x-\beta x^{\gamma}}\right\}-1}\right\}, x>0 .
$$

Proposition 2.24. Let $X: \Omega \rightarrow(0, \infty)$ be a continuous random variable. The random variable $X$ has $c d f(74)$ if and only if its hazard function $h_{F}(x)$ satisfies the differential equation

$$
h_{F}^{\prime}(x)-\frac{g^{\prime}(x ; \Theta)}{g(x ; \Theta)} h_{F}(x)=\alpha g(x ; \Theta) \times \frac{d}{d x}\left\{\frac{\alpha e^{\gamma(-\log \bar{G}(x ; \Theta))}(\gamma(-\log \bar{G}(x ; \Theta))+1)+\lambda e^{\lambda(-\log \bar{G}(x ; \Theta))-\beta}}{\bar{G}(x ; \Theta)}\right\}, x>0 .
$$

Proposition 2.25. Let $X: \Omega \rightarrow(0, \infty)$ be a continuous random variable. The random variable $X$ has $c d f$ (75) if and only if its hazard function $h_{F}(x)$ satisfies the differential equation

$$
\begin{aligned}
& h_{F}^{\prime}(x)+\lambda \beta x^{\beta-1} h_{F}(x)=\alpha \beta \theta \lambda^{2} e^{-\lambda x^{\beta}} \times \\
& \frac{d}{d x}\left\{\frac{x^{\beta-1}\left(1+x^{\beta}\right) e^{-\lambda x^{\beta}}\left[1-\left(1+\frac{\lambda x^{\beta}}{\lambda+1}\right) e^{-\lambda x^{\beta}}\right]^{\alpha-1} C^{\prime}\left(\theta-\theta\left[1-\left(1+\frac{\lambda x^{\beta}}{\lambda+1}\right) e^{-\lambda x^{\beta}}\right]^{\alpha}\right)}{C\left(\theta-\theta\left[1-\left(1+\frac{\lambda x^{\beta}}{\lambda+1}\right) e^{-\lambda x^{\beta}}\right]^{\alpha}\right)}\right\}, x>0 .
\end{aligned}
$$

Proposition 2.26. Let $X: \Omega \rightarrow \mathbb{R}$ be a continuous random variable. The random variable $X$ has $c d f$ (76) if and only if its hazard function $h_{F}(x)$ satisfies the differential equation

$$
h_{F}^{\prime}(x)-\frac{f_{R}^{\prime}(x)}{f_{R}(x)} h_{F}(x)=\frac{c}{\gamma^{c}} \frac{d}{d x}\left\{\frac{\left[-\log \left(1-F_{R}(x)\right)\right]^{c-1}}{1-F_{R}(x)}\right\}, x \in \mathbb{R} .
$$

Proposition 2.27. Let $X: \Omega \rightarrow(0, \infty)$ be a continuous random variable. The random variable $X$ has $c d f(79)$ if and only if its hazard function $h_{F}(x)$ satisfies the differential equation

$$
h_{F}^{\prime}(x)-\frac{\beta-1}{x} h_{F}(x)=\frac{\beta x^{\beta-1}}{(\gamma(\mathbf{t}))^{\beta}} \frac{d}{d x}\left\{\frac{1-\lambda+2 \lambda \exp \left[-\left(\frac{x}{\gamma(\mathbf{t})}\right)^{\beta}\right]}{1-\lambda+\lambda \exp \left[-\left(\frac{x}{\gamma(\mathbf{t})}\right)^{\beta}\right]}\right\}, x>0 .
$$

\subsection{Characterization in Terms of the Reverse (or Reversed) Hazard Function}

The reverse hazard function, $r_{F}$, of a twice differentiable distribution function, $F$, is defined as $r_{F}(x)=\frac{f(x)}{F(x)}, x \in \operatorname{support}$ of $F$. In this subsection we present characterizations of ETWG (for $\lambda=0$ ), GEG, ELG (for $\alpha=1$ ), KwTP (for $b=1$ ), MOEIP, SSTE, GBXIIP, OLBXII, MWP, EPLG distributions in terms of the reverse hazard function. 
Proposition 3.1. Let $X: \Omega \rightarrow(0, \infty)$ be a continuous random variable. The random variable $X$ has $p d f(4)$ (for $\lambda=0$ ) if and only if its reverse hazard function $r_{F}(x)$ satisfies the following differential equation

$$
r_{F}^{\prime}(x)+\frac{\beta x^{\beta-1}}{\sigma^{\beta}} r_{F}(x)=\frac{\alpha \beta(1-p)}{\sigma} e^{-\left(\frac{x}{\sigma}\right)^{\beta}} \frac{d}{d x}\left\{\frac{\left(\frac{x}{\sigma}\right)^{\beta-1}}{\left[1-e^{-\left(\frac{x}{\sigma}\right)^{\beta}}\right]\left\{1-p+p\left[1-e^{-\left(\frac{x}{\sigma}\right) \beta}\right]^{\alpha}\left[1+\lambda e^{-\left(\frac{x}{\sigma}\right) \beta}\right]^{\alpha}\right\}}\right\}, x>0 .
$$

Proof. If $X$ has $p d f$ (4) for $\lambda=0$, then clearly the above differential equation holds. If the differential equation holds, then

$$
\frac{d}{d x}\left\{e^{\left(\frac{x}{\sigma}\right) \beta} r_{F}(x)\right\}=\frac{\alpha \beta(1-p)}{\sigma} \frac{d}{d x}\left\{\frac{\left(\frac{x}{\sigma}\right)^{\beta-1}}{\left[1-e^{-\left(\frac{x}{\sigma}\right)^{\beta}}\right]\left\{1-p+p\left[1-e^{-\left(\frac{x}{\sigma}\right) \beta}\right]^{\alpha}\left[1+\lambda e^{-\left(\frac{x}{\sigma}\right) \beta}\right]^{\alpha}\right\}}\right\},
$$

from which we arrive at the hazard function corresponding to the $c d f(2)$.

A Proposition similar to that of Proposition 3.1 will be stated (without proof) for each one of the distributions listed in subsection 3.1 .

Proposition 3.2. Let $X: \Omega \rightarrow \mathbb{R}$ be a continuous random variable. The random variable $X$ has $c d f(7)$ if and only if its reverse hazard function $r_{F}(x)$ satisfies the following differential equation

$$
r_{F}^{\prime}(x)-\alpha g(x)(G(x))^{\alpha-1} r_{F}(x)=\alpha \exp \left\{(G(x))^{\alpha}\right\} \frac{d}{d x}\left\{\frac{g(x)(G(x))^{\alpha-1}}{\exp \left\{(G(x))^{\alpha}\right\}-1}\right\}, x \in \mathbb{R} .
$$

Proposition 3.3. Let $X: \Omega \rightarrow(0, \infty)$ be a continuous random variable. The random variable $X$ has $p d f(20)$, for $\alpha=1$, if and only if its reverse hazard function $r_{F}(x)$ satisfies the following differential equation

$$
r_{F}^{\prime}(x)+\lambda(1+\lambda x)^{-1} r_{F}(x)=(1-p) \lambda \theta(1+\lambda x)^{-(\theta+1)} \frac{d}{d x}\left\{\frac{\left[1-(1+\lambda x)^{-\theta}\right]^{-1}}{1-p(1+\lambda x)^{-\theta}}\right\}, x>0 .
$$

Proposition 3.4. Let $X: \Omega \rightarrow(0, \infty)$ be a continuous random variable. The random variable $X$ has $c d f(19)$, for $b=1$, if and only if its reverse hazard function $r_{F}(x)$ satisfies the following differential equation

$$
r_{F}^{\prime}(x)+\frac{\alpha+1}{x} r_{F}(x)=a b \alpha \theta^{\alpha} x^{-(\alpha+1)} \frac{d}{d x}\left\{\frac{\left[1-\lambda+2 \lambda\left(\frac{\theta}{x}\right)^{\alpha}\right]}{\left[1-\left(\frac{\theta}{x}\right)^{\alpha}\right]\left[1+\lambda\left(\frac{\theta}{x}\right)^{\alpha}\right]}\right\}, \quad x>0 .
$$

Proposition 3.5. Let $X: \Omega \rightarrow(0, \infty)$ be a continuous random variable. The random variable $X$ has $c d f(21)$, if and only if its reverse hazard function $r_{F}(x)$ satisfies the following differential equation

$$
r_{F}^{\prime}(x)-\frac{\alpha-1}{x+\beta} r_{F}(x)=\alpha \beta \delta(x+\beta)^{\alpha-1} \frac{d}{d x}\left\{\frac{1}{x\left[\delta(x+\beta)^{\alpha}-(\delta-1) x^{\alpha}\right]}\right\}, x>0 .
$$

Proposition 3.6. Let $X: \Omega \rightarrow(0, \infty)$ be a continuous random variable. The random variable $X$ has $c d f(29)$, if and only if its reverse hazard function $r_{F}(x)$ satisfies the following differential equation

$$
r_{F}^{\prime}(x)+\theta r_{F}(x)=-\frac{\pi}{2} \theta^{2} e^{-2 \theta x}\left[1+\tan ^{2}\left(\frac{\pi}{2} e^{-\theta x}\right)\right], \quad x>0 .
$$

Proposition 3.7. Let $X: \Omega \rightarrow(0, \infty)$ be a continuous random variable. The random variable $X$ has $c d f(30)$, if and only if its reverse hazard function $r_{F}(x)$ satisfies the following differential equation

$$
r_{F}^{\prime}(x)-\frac{\alpha-1}{x} r_{F}(x)=a^{2} \alpha \beta(\beta+1) \lambda x^{2(\alpha-1)}\left(1+x^{\alpha}\right)^{-\beta-2}+a \alpha \beta \lambda x^{\alpha-1} \frac{d}{d x}\left\{\frac{\left(1+x^{\alpha}\right)^{-\beta-1}}{1-\exp \left(\lambda\left(\left(1+x^{\alpha}\right)^{-\beta}-1\right)\right)}\right\}, x>0 .
$$


Proposition 3.8. Let $X: \Omega \rightarrow(0, \infty)$ be a continuous random variable. The random variable $X$ has $c d f(65)$, if and only if its reverse hazard function $r_{F}(x)$ satisfies the following differential equation

$$
r_{F}^{\prime}(x)-\frac{\alpha-1}{x} r_{F}(x)=\theta \alpha \beta \sigma^{-\alpha} x^{\alpha-1} \frac{d}{d x}\left\{\frac{\left[1+\left(\frac{x}{\sigma}\right)^{\alpha}\right]^{2 \beta-1}}{1+\theta\left[1+\left(\frac{x}{\sigma}\right)^{\alpha}\right]^{\beta}}\right\}, x>0 .
$$

Proposition 3.9. Let $X: \Omega \rightarrow(0, \infty)$ be a continuous random variable. The random variable $X$ has $c d f(71)$, if and only if its reverse hazard function $r_{F}(x)$ satisfies the following differential equation

$$
r_{F}^{\prime}(x)+\left(\alpha+\beta \gamma x^{\gamma-1}\right) r_{F}(x)=\theta e^{-\alpha x-\beta x^{\gamma}} \frac{d}{d x}\left\{\frac{\alpha x+\beta \gamma x^{\gamma-1}}{1-\exp \left\{-\theta\left(1-e^{-\alpha x-\beta x^{\gamma}}\right)\right\}}\right\}, x>0
$$

Proposition 3.10. Let $X: \Omega \rightarrow(0, \infty)$ be a continuous random variable. The random variable $X$ has $c d f(72)$, if and only if its reverse hazard function $r_{F}(x)$ satisfies the following differential equation

$$
r_{F}^{\prime}(x)+\lambda \beta x^{\beta-1} r_{F}(x)=\frac{\alpha \beta \lambda^{2}}{\lambda+1} e^{-\lambda x^{\beta}} \times \frac{d}{d x}\left\{\frac{x^{\beta-1}\left(1+x^{\beta}\right)\left[1-\left(1+\frac{\lambda x^{\beta}}{\lambda+1}\right) e^{-\lambda x^{\beta}}\right]^{-1}}{1-\theta\left[1-\left(1+\frac{\lambda x^{\beta}}{\lambda+1}\right) e^{-\lambda x^{\beta}}\right]^{\alpha}}\right\}, x>0 .
$$

\subsection{Characterization Based on the Conditional Expectation of Certain Function of the Random Variable}

In this subsection we employ a single function $\psi$ (or $\psi_{1}$ ) of $X$ and characterize the distribution of $X$ in terms of the truncated moment of $\psi(X)$ (or $\psi_{1}(X)$ ). The following propositions have already appeared in Hamedani's previous work (2013), so we will just state them here which can be used to characterize some of the distributions listed in Section 1.

Proposition H1. Let $X: \Omega \rightarrow(e, f)$ be a continuous random variable with $c d f F$. Let $\psi(x)$ be a differentiable function on $(e, f)$ with $\lim _{x \rightarrow e^{+}} \psi(x)=1$. Then for $\delta \neq 1$,

$$
E[\psi(X) \mid X \geq x]=\delta \psi(x), \quad x \in(e, f),
$$

if and only if

$$
\psi(x)=(1-F(x))^{\frac{1}{\delta}-1}, \quad x \in(e, f) .
$$

Proposition H2. Let $X: \Omega \rightarrow(e, f)$ be a continuous random variable with $c d f F$. Let $\psi_{1}(x)$ be a differentiable function on $(e, f)$ with $\lim _{x \rightarrow f^{-}} \psi_{1}(x)=1$. Then for $\delta_{1} \neq 1$,

$$
E\left[\psi_{1}(X) \mid X \leq x\right]=\delta_{1} \psi_{1}(x), \quad x \in(e, f),
$$

implies

$$
\psi_{1}(x)=(F(x))^{\frac{1}{\delta_{1}}-1} . \quad x \in(e, f) .
$$

Remarks 4.1. (A) For $(e, f)=(0, \infty), \psi(x)=1-\exp \left\{-a(1+\eta x)^{-1 / \eta}\right\}$ and $\delta=\frac{b}{1+b}$, Proposition H1 provides a characterization of KumGEV distribution. $(B)$ For $(e, f)=(0, \infty), \psi(x)=\exp \left\{-\left(\log \left[1+\left(\frac{x}{s}\right)^{u}\right]\right)^{\beta}\right\}$ and $\delta=\frac{\alpha}{1+\alpha}$, Proposition $\mathrm{H} 1$ provides a characterization of GWBXII distribution. $(C)$ For $(e, f)=(0, \infty), \psi(x)=\exp \left\{-\frac{\left(1-e^{-(\lambda x)^{2}}\right)^{\theta \beta}}{\left[1-\left(1-e^{-(\lambda x)^{2}}\right)^{\theta}\right]^{\beta}}\right\}$ and $\delta=\frac{\alpha}{1+\alpha}$, Proposition H1 provides a characterization of WBX distribution. $(D)$ For $(e, f)=(0, \infty), \psi(x)=$ $1-\left[1-\left(\frac{\theta}{x}\right)^{\alpha}\right]^{a}\left[1+\lambda\left(\frac{\theta}{x}\right)^{\alpha}\right]^{a}$ and $\delta=\frac{b}{1+b}$, Proposition H1 provides a characterization of KwTP distribution. (E) For $(e, f)=(0, \infty), \lambda=0, \psi(x)=\left(1-e^{-\left(\frac{\theta}{x}\right)^{\beta}}\right)$ and $\delta=\frac{\alpha}{1+\alpha}$, Proposition H1 provides a characterization of TEF distribution. $(F)$ For $(e, f)=(0, \infty), \psi(x)=\frac{1-\exp \left(\lambda\left(\left(1+x^{\alpha}\right)^{-\beta}-1\right)\right)}{1-e^{-\lambda}}$ and $\delta=\frac{a}{1+a}$, Proposition $\mathrm{H} 2$ provides a characterization of GBXIIP distribution. $(G)$ For $(e, f)=(0, \infty), \lambda=0, \psi(x)=\left(\frac{\theta+\alpha+\alpha \theta x}{\theta+\alpha}\right)^{1 / \theta} e^{-x}$ and $\delta=\frac{\theta}{1+\theta}$, Proposition H1 provides a characterization of TLL distribution. $(H)$ For $(e, f)=(0, \lambda), \psi(x)=\left[1-\left(\frac{x}{\lambda}\right)^{a \theta}\right]$ and $\delta=\frac{b}{1+b}$, Proposition H1 
provides a characterization of Kw-PF distribution. (I) For $(e, f)=\mathbb{R}, \psi(x)=\left(1+(-\log (\bar{G}(x ; \eta)))^{c}\right)^{-1}$ and $\delta=\frac{k}{1+k}$ , Proposition H1 provides a characterization of GBG distribution. (J) For $(e, f)=(0, \infty), \psi(x)=\exp \left\{-\left[e^{\lambda x^{\gamma}}-1\right]^{\beta}\right\}$ and $\delta=\frac{\alpha}{1+\alpha}$, Proposition H1 provides a characterization of WW distribution. $(K)$ For $(e, f)=(0,1), \psi(x)=e^{x^{\alpha}}$ and $\delta=\frac{\ln 2}{1+\ln 2}$, Proposition H1 provides a characterization of NLM distribution. $(L)$ For $(e, f)=(0, \infty), \psi(x)=$ $\left(\frac{1+\theta\left[1+\left(\frac{x}{\sigma}\right)^{\alpha}\right]^{\beta}}{1+\theta}\right)^{1 / \theta} \exp \left\{\left(1-\left[1+\left(\frac{x}{\sigma}\right)^{\alpha}\right]^{\beta}\right)\right\}$ and $\delta=\frac{\theta}{1+\theta}$, Proposition H1 provides a characterization of OLBXII distribution. (M) For $(e, f)=(0, \infty), \psi(x)=\left(1+x^{c}\right)^{-1} e^{-\frac{\alpha}{k} x^{\beta} e^{\lambda x}}$ and $\delta=\frac{k}{1+k}$, Proposition H1 provides a characterization of BXIIMW distribution. $(N)$ For $(e, f)=\mathbb{R}, \psi(x)=\exp \left\{-\left[-\log \left(1-F_{R}(x)\right)\right]^{c \gamma^{c}}\right\}^{1 / \gamma^{c}}$ and $\delta=\frac{1}{1+\gamma^{c}}$, Proposition H1 provides a characterization of W-R distribution.

\section{Infinite Divisibility}

Bondesson (1979) showed that all the members of the following families

$$
\begin{gathered}
f(x)=C x^{\beta-1}\left(1+c x^{\alpha}\right)^{-\gamma}, \quad x>0, \quad 0<\alpha \leq 1, \\
f(x)=C x^{\beta-1} \exp \left\{-c x^{\alpha}\right\}, \quad x>0, \quad 0<|\alpha| \leq 1, \\
f(x)=C x^{\beta-1} \exp \left\{-\left(c_{1} x+c_{2} x^{-1}\right)\right\}, \quad x>0,-\infty<\beta<\infty, \\
f(x)=C x^{-1} \exp \left\{-(\log x-\mu)^{2} /\left(2 \sigma^{2}\right)\right\}, \quad x>0,
\end{gathered}
$$

where the natural restrictions are put on the unspecified parameters, are infinitely divisible. The last one is the lognormal density.

Remark 3.1. Bondesson (1992, Theorem 6.2.4) pointed out that multiplying densities $\left(B_{1}\right)-\left(B_{4}\right)$ by $C_{1}(\delta+x)^{-v}$ for $\delta>0$ and $v>0$, will result in densities which are also infinitely divisible.

Remark 3.2. The distributions, listed in Section 1, whose densities can be expressed, in view of Remark 3.1, in the form $\left(B_{1}\right)$ are: BTXII , ZBXII (for $a=1$ ), MOEIP (for $\delta=1$ ), LLGW and MB-F (for $a+c>1$ ); in the form $\left(B_{2}\right)$ are: EBXII (for $\beta=v=b=1,|\alpha| \leq 1$ ), McQL (for $a=b=1$ ); in the form of $\left(B_{3}\right)$ are: TIGE for $\alpha=1$, TGIE (for $\alpha=1, \lambda=0$ ), MOEIW (for $\theta=\beta=1$ ) and TEF (for $\alpha=\beta=1, \lambda=0$ ).

\section{Concluding Remarks}

In designing a stochastic model for a particular modeling problem, an investigator will be vitally interested to know if their model fits the requirements of a specific underlying probability distribution. To this end, the investigator will vitally depend on the characterizations of the selected distribution. A good number of recently introduced distributions which have important applications in many different fields have been mentioned in this work. Certain characterizations of these distributions have been established. We hope that these results will be of interest to the investigators who may believe their models have distributions mentioned here and are looking for justifying the validity of their models. It is known that determining a distribution is infinitely divisible or not via the existing representations is not easy. We have used Bondesson's classifications to show that some of the distributions taken up in this work are infinitely divisible. This could be helpful to some researchers. Finally, we like to mention that the distributions mentioned in this work may be a source of preventing duplications of the existing distributions.

\section{References}

Abdelall, Y. Y. (2016). The odd generalized exponential modified Weibull distribution. International Mathematical Forum, 11(19), 943-959.

Abd El-Monsef, M. M. E., Hassanein, W. A., \& Kilany, N. M. (2017). Erlang-Lindley distribution. Commun. Statist. Theo-Meth., 46(19), 9494-9506.

Abdel-Hamid, A. H., \& Albasuoni, N. A. (2016). Log-logistic generated Weibull distribution: Model, properties, applications and estimations under progressive type-II censoring. J. Stat. Appl. Pro., 5(2), 221-232.

Abdul-Moniem, I. B. (2017). The Kumarasamy power function distribution. J. Stat. Appl. Pro., 6(1), 81-90. 
Abouelmagd, T. H. M., Hamed, M. S., \& Afify, A. Z. (2017). The extended Burr XII distribution with variable shape for the hazard rate. Pak.j.stat.oper.res., XIII(1), 687-698.

Abouelmagd, T. H. M., Hamed, M. S., \& Ebraheim, A. E. H. N. (2017). The Poisson-G family of distributions with applications. Pak.j.stat.oper.res., XIII(2), 313-326.

Abouelmagd, T. H. M., Al-mualim, S., Elgarhy, M., Afify, A. Z., \& Ahmad, M. (2017). Properties of the four-parameter Weibull distribution and its applications. Pak.J.Statist., 33(6), 449-466.

Abouelmagd, T. H. M., Al-mualim, S., Afify, A. Z., Ahmad, M., \& Al-Mofleh, H. (2018). The odd Lindley Burr XII distribution with applications. Pak.J.Statist., 34(1), 15-32.

Afify, A. Z., Cordeiro, G. M., Ortega, E. M. M., Yousof, H. M., \& Butt, N. S. (2017). The four parameter Burr XII distribution: Properties, Regression model and Applications, Commun. Statist. Theo-Meth.

Afify, A. Z., Yousof, H. M., Hamedani, G. G., \& Aryal, G. (2016). The exponentiated Weibull-Pareto distribution with application. J. of Statistical Theory and Applications (JSTA), 15, 326-344.

Ahmad, A., Ahmad, S. P., \& Ahmed, A. (2015). Characterization and estimation of transmuted Rayleigh distribution. $J$. Stat. Appl. Pro., 4(2), 315-321.

Ahsan ul Haq, M. (2016). Transmuted exponentiated inverse Rayleigh distribution. J. Stat. Appl. Pro., 5(2), 337-343.

Ahsan ul Haq, M., Yousof, H. M., \& Hashmi, S. (2017). A new five-parameter Fréchet model for extreme values, Pak.j.stat.oper.res. XIII(1), 617-632.

Alizadeh, M., Bagheri, S. F., Alizadeh, Mohammad and Nadarajah, S. (2016). A new four-parameter lifetime distribution. J. of Applied Statistics.

Alizadeh, M., Bagheri, S. F., Bahrami Samani, Ghobagi, S., \& Nadarajah, S. Exponentiated power Lindley power series class of distributions: theory and applications. (Forthcoming).

Alizadeh, M., Cordeiro, G. M., de Brito, E., \& Demétrio, C. G. (2017). The beta Marshall-Olkin family of distributions. JSDA, 2, 1-18.

Alizadeh, M., MirMostafee, S. m. T. k., Ortega, E. M. M., Ramires, T. G., \& Cordeiro, G. M. (2017). The odd log-logistic Logarithmic generated family of distributions with applications in different areas. JSDA, 4:6, 1-25.

Al-babtain, A., Fattah, A. A., Ahmed, A-hadi N., \& Merovci, F. (2017). Kumaraswamy-transmuted exponentiated modified Weibull distribution, Commun. Statist. Simulation and Computation, 2015 online; In print on Jan. 25, 38123832 .

Al-Khazaleh, A. M. H. (2016). Transmuted Burr Type XII distribution: A generalization of the Burr Type XII distribution. International Mathematical Forum, 11(12), 547-556.

Al-Khazaleh, M., Al-Omari, A. I., \& Al-Khazaleh, A. M. H. (2016). Transmuted two-parameter Lindley distribution. $J$. Stat. Appl. Pro., 5(3), 421-432.

Al-Omari, A. I., \& Al-Khazaleh, A. M. H., \& Alzoubi, L. M. (2017). Transmuted Janardan distribution: a generalization of the Janardan distribution. J. Stat. Appl. Pro., 5(2), 259-269.

Alzaatreh, A., Famoye, F., \& Lee, C. (2012). Gamma-Pareto distribution and its application. J. Modern Applied Statistical Methods, 11, 78-94.

Arifa, S., Yab, M. Z., \& Ali, A. (2017). The modified Burr III G family of distributions. JDS, 15(1), 41-60.

Asgharzadeh, A., Nadarajah, S., \& Sharafi, F. (2016). Generalized inverse Lindley distribution with application to Danish fire insurance data, Commun. in Statist. Theo-Meth. 45, 5001-5021.

Badr, A. M. M. (2017). Transmuted Kumaraswamy exponentiated inverse Rayleigh distribution. Mathematical Theory and Modeling, 7(3), 41-55.

Bagheri, S. F., Bahrami Samani, E., \& Ganjali, M. (2016). The generalized modified Weibull power series distribution: theory and applications. Computational Statistics and Data Analysis, 94, 136-160.

Barco, K. V., Mazucheli, J., \& Janeiro, V. (2017). The inverse power Lindley distribution. Commun. in Statist. Simulation and Computation, 46, 6308-6323.

Bourguignon, M., Silva, R. B., Zea, L. M. Zea Cordeiro, G. M. (2013). The Kumaraswamy Pareto distribution. J. Stat. Theory \& Appl., 12, 129-144. 
Brito, E., Cordeiro, G. M., Yousof, H. M., Alizadeh, M., \& Silva, G. O. (2017). The Topp-Leone odd log-logistic family of distributions. J. Statistical Computation and Simulation (JSCS), 87(15), 3040-3058.

Bulut, Y. M., Jamalizadeh, A., Dogru, F. Z., \& Arslan, O. (2017). The generalized half-t distribution. Statistics and Its Inference, 10, 727-734.

Chakraborty, S., \& Handique, L. (2017). The generalized Marshall-Olkin-Kumaraswamy-G family of distributions. Journal of Data Science, 15, 391-422.

Chhetri, S. B., Akinsete, A. A., Aryal, G., \& Long, H. (2017). The Kumaraswamy transmuted Pareto distribution. J. Statistical Distributions and Applications (JSDA), 4:11, 1-24.

Chhetri, S. B., Long, H., \& Aryal, G. (2017). The beta transmuted Pareto distribution: Theory and Applications. J. Stat. Appl. Pro., 6(2), 243-258.

Condino, F., \& Domma, F. A new distribution function with bounded support: the reflected generalized Topp-Leone power series distribution, (METRON, forthcoming).

Cordeiro, G. M., Alizadeh, M., Ortega, E. M. M., \& Serrano, L. H. V. (2016). The Zografos-Balakrishnan odd log-logistic family of distributions: properties and Applications. HJMS, 45, 1781-1803.

Cordeiro, G. M., Alizadeh, M., Silva, R. B., \& Ramires, T. G. (To appear, 2016). A new wider family of continuous models: The extended Cordeiro and de Castro family.

Cordeiro, G. M., Lima, M. C. S., Gomes, A. Z., da Silva, C. O., \& Ortega, E. M. M. (2016). The gamma extended Weibull distribution. J. Statistical Distributions and Applications, 3, 1-18.

Cordeiro, G. M., Mead, M. E., Afify, A. Z., Suzki, A. K., \& Abd El-Gaied, A. A. K. (2017). An extended Burr XII distribution: Properties, Inference and Applications. PJSOR, XIII(\$), 809-828.

Cordeiro, G. M., Nadarajah, S., Ortega, E. M. M., \& Ramires, T. G. An alternative two-parameter gamma generated family of distributions: properties and applications. (To appear in HJMS).

Cordeiro, G. M., Ortega, E. M. M., \& da Cunha, D. C. C. (2013). The exponentiated generalized class of distributions. J. of Data Science, 11, 1-27.

Dar, A. A., Ahmed, A., \& Reshi, J. A. (2017). Transmuted weighted exponential distribution and its application. J. Stat. Appl. Pro., 6(1), 219-232.

Dey, S., Dey, T., Ali, S., \& Mulekar, M. S. (2016). Two-parameter Maxwell distribution: properties and different methods of estimation. J. of Statistical Theory and Practice (JSTP), 10(2), 291-310.

Dey, S., Nassar, M., \& Kumar, D. (2017). $\alpha$ Logarithmic transformed family of distributions with application. ADS (Annals of Data Science), 4(4), 457-458.

Dias, C. R. B., Alizadeh, M., \& Cordeiro, G. M. The beta Nadarajah-Haghighi distribution, (HJMS, forthcoming).

Dias, C. R. B., Cordeiro, G. M., Alizadeh, M., Diniz Marinho, P. R., \& Campos Coêlho, H. F. (2016). Exponentiated Marshall-Olkin family of distributions. JSDA, 3:15, 1-21.

El-Bassiouny, A. H., El-Damcese, M., Mustafa, A., \& Eliwa, M. S. (2017). Exponentiated generalized Weibull-Gompertz distribution with application in survival analysis. J. Stat. Appl. Pro., 6(1), 7-16.

Elgarhy, M. On the exponentiated Weibull Rayleigh distribution. (Personal Communication).

Elbatal, I. (2013). Transmuted generalized inverted exponential distribution. Econ. Qual. Control, 26(2), $125-133$.

Elbatal, I., \& Aryal, G. On the transmuted additive Weibull distribution. Austrian J. of Statistics, 42 (2013), 117 - 132.

Elbatal, I., Asha, G., \& Raja, A. V. (2014). Transmuted exponentiated Fréchet distribution: Properties and Applications. J. Stat. Appl. Pro., 3(3), 379-394.

Elbatal, I., \& Elgarhy, M. (2013). Statistical properties of Kumaraswamy Quasi Lindley distribution. International J. of Mathematics Trend and Technology, 4, 237-246.

Elbatal, I., El Gebaly, Y. M., \& Amin, E. A. (2017). The beta generalized inverse Weibull geometric distribution and its applications, Pak.j.stat.oper.res. XIII(1), 75-90.

Elbatal, I., Zayed, M., Rasekhi, M., \& Butt, N. S. (2017). The Exponential Pareto power series distribution: Theory and Applications, Pak.j.stat.oper.res. XIII(1), 603-615. 
El-Damcese, M. A., Mustafa, A., El-Desouky, B. S., \& Mustafa, M. E. (2016). The odd generalized exponentiated linear failure rate distribution. J. Stat. Appl. Pro., 5(2), 299-309.

Elgarhy, M. On the exponentiated Weibull Rayleigh distribution. (Personal Communication).

Elgarhy, M., Shakil, M., \& Kibria, B. M. G. (2017). Exponentiated Weibull-exponential distribution with applications, AAM (Applications and Applied Mathematics), 12(2), 710-725.

El-Gohary, A., El-Bassiouny, A. H., \& El-Morshedy, M. (2015). Inverse flexible Weibull extension distribution. International J. of Computer Applications, 115 46-51.

Eljabri, S., \& Nadarajah, S. (2017). The Kumaraswamy GEV distribution. Commun. Statist. Theo-Meth., 46(20), 1020310235.

El-Morshedy, M., El-Bassiouny, A. H, \& El-Gohary, A. (2017). Exponentiated inverse flexible Weibull extension distribution. J. Stat. Appl. Pro., 6(1), 169-183.

Fattah, A. A., Nadarajah, S., \& Ahmad, A. N. (2017). The exponentiated transmuted Weibull geometric distribution with application in survival analysis. Commun. Statist. Simul. Comp., 46(6), 4244-4263.

Genç, A. (2017). Truncated inverted generalized exponential distribution and its properties. Commun. Statist. Simul. Comp. (CSSC), 46(6), 4654-4670.

Gharib, M., Mohammed, B. I., \& Aghel, W. E. R. (2017). Marshall-Olkin extended inverse Pareto distribution and its application. IJSP, 6(6), 71-84.

Ghorbani, M., Bagheri, S. F., \& Alizadeh, M. (2014). A new lifetime distribution: The modified Weibull Poisson distribution. International journal of Operational Research and Decision Science Studies, 1(2), 28-47.

Ghorbani, M., Bagheri, S. F., \& Alizadeh, M. (2017). A new family of distributions: The additive modified Weibull odd log-logistic-G Poisson family, properties and applications. Ann. Data Sci., 4(2), 249-287.

Ghosh, I., \& Nadarajah, S. On some further properties and application of Weibull-R family of distributions, Annals of Data Science. (Forthcoming).

Glänzel, W. (Bad Tatzmannsdorf, 1986). A characterization theorem based on truncated moments and its application to some distribution families, Mathematical Statistics and Probability Theory, Vol. B, Reidel, Dordrecht, $1987,75-84$.

Glänzel, W. (1990). Some consequences of a characterization theorem based on truncated moments. Statistics: A Journal of Theoretical and Applied Statistics, 21(4), 613-618.

Glänzel, W., \& Hamedani, G. G. (2001). Characterizations of univariate continuous distributions. Studia Sci. Math. Hungar., 37, 83-118.

Gomez-Silva, F. S., Percontini, A., de Brito, E., Ramos, M. W., Venancio, R., \& Coreiro, G. M. (2017). The odd Lindley-G family of distributions. Austrian J. Stat., 49(1), 57-79.

Granzotto, D. C. T., Santos, C. A., \& Louzada, F. (2018). The transmuted Weibull regression model: an application to type 2 diabetes Mellitus data. IJSP, 7(2), 1-11.

Guerra, R. R., Peña-Ramirez, S. A., \& Cordeiro, G. M. (2017). The gamma Burr XII distribution: Theory and application. Journal of Data Science, 15, 467-494.

Hamedani, G.G. (2016). On characterizations and infinite divisibility of recently introduced distribution. SMH, 53(4), 467-511.

Hamedani, G. G. (2013). On certain generalized gamma convolution distributions II, Technical Report No. 484. MSCS, Marquette University.

Hamedani, G. G. (2018). Characterizations and infinite divisibility of certain 2016-2017 univariate continuous distributions III. IJSP, 7(1), 39-71.

Hamedani, G. G. (2017a). Characterizations and infinite divisibility of certain 2016-2017 univariate continuous distributions II. IMF (International Mathematical Forum), 12(12), 565-609.

Hamedani, G. G., \& Maadooliat, M. (Research Monograph, 2017). Characterizations of recently introduced univariate continuous distributions.

Hamedani, G. G., \& Safavimanesh, F. (2017b). Characterizations and infinite divisibility of certain 2016 univariate continuous distributions. IMF, 12(5), 195-228. 
Handique, L., Chakraborty, S., \& Ali, M. M. (2017). Beta generated Kumarswamy-G family of distributions. Pak.J.Statist., 33(6), 467-490.

Hassan, A. S., \& Abd-Allah, M. (2017a). Exponentiated Lomax geometric distribution: properties and Applications. Pak.j.stat.oper.res., XIII(2), 545-566.

Hassan, A. S., \& Assar, S. M. (2017). The exponentiated Weibull-Power Function distribution. JDS, 15, 589-614.

Hassan, A. S., \& Nssar, S. G. (2018). Power Lomax Poisson distribution: properties and estimation. JDS, 18, 105-128.

Ibrahim, N. A., Khaleel, M. A., Merovci, F., Kilicman, A., \& Shitan, M. (2017). Weibull Burr X distribution: Properties and Application. Pak.J.Statist., 33(5), 315-336.

Jamal, F., Nasir, M. A., Tahir, M. H., \& Montazeri, N. H. (2017). The odd Burr-III family of distributions. J. Stat. Appl. Pro., 6(1), 105-122.

Joshi, S., \& Jose, K. K. (2018). Wrapped Lindley distribution. CSTM, 47(5), 1013-1021.

Kemaloglu, S. A., \& Yilmaz, M. (2017). Transmuted two-parameter Lindley distribution. CSTM, 46(23), 11866-11879.

Khan, M. S., King, R., \& Hudson, I. L. (2017a). Transmuted new generalized inverse Weibull distribution. Pak.j.stat.oper.res., $\operatorname{XIII}(2), 227-296$.

Khan, M. S., King, R., \& Hudson, I. L. (2017b). Transmuted generalized exponential distribution: A generalization of the exponential distribution with applications to survival data. Commun. Statist. Simul. Comp., 46(6), 4377-4398.

Korkmaz, M. C., Altun, E., Yousof, H. M., Afify, A. Z., \& Nadarajah, S. (2018). The Burr X Pareto distribution: properties, application and VaR estimate. J. of Risk and Financial Management, 11(1), 1-16.

Korkmaz, M. C., \& Yousof, M. H. (2017). The one-parameter odd Lindley exponential model: Mathematical properties and Applications. Stochastics and Quality Control, 32(1), 25-35.

Krishna, E., Jose, K. K., \& Ristić, M. M. (2013). The Marshall-Olkin Fréchet distribution. Communications in Statistics: Theory and Methods, 42, 4091-4107.

Kumar, D. (2017). The Burr Type XII distribution with some statistical properties. Journal of Data Science, 16(3), 509-534.

Kumar, D., Singh, U., \& Singh, S. K. (2015). A new distribution using sine function-its application to bladder cancer patient data. J. Stat. Appl. Pro., 4(3), 417-427.

Lepetu, L., Oluyede, B. O., Makubate, B., Foya, S., \& Mdlongwa, P. (2017). Marshall-Olkin log-logistic extended Weibull distribution: Theory, Properties and Applications. JDS, 15, 691-722.

Luguterah, A., \& Nasiru, S. (2015). The odd generalized exponential generalized linear exponential distribution. J. Stat. Appl. Pro., 6(1), 139-148.

Mahmoud, M. R., El-Sherpieny, E. A., \& Ahmad, M. A. (2015). The new Kumaraswamy Kumaraswamy family of distributions with application. PJSOR, 11, 159-180.

Maksaei, N., \& Altun, E. (2017). The generalized Weibull-Burr XII distribution and its applications. Journal of Data Science, 16(3), 535-552.

Makubate, B., Oluyede, B.O., Motobesto, G., Haung, S., \& Fagbamigbe, A. F. (2018). The beta Weibull-G family of distributions: model, properties and application. IJSP, 7(2), 12-32.

Mansoor, M., Tahir, M., Alizadeh, M., Cordeiro, G. M., Zubair, M., \& Ghazali, S. S. A. (2016). An extended Fréchet distribution: Properties and Applications. Journal of Data Science, 14, 167-188.

Mansour, M. M., \& Abd Elrazik, E. M. (2016). A new distribution to analyze a practical problem with applications. $J$. Stat. Appl. Pro., 5(3), 481-494.

Maurya, S. K., Kaushik, A., Singh, S. K. \& Singh, U. (2017). A new class of distribution having decreasing, increasing and bathtub-shaped failure rate. Commun. Statist. Theo-Meth., 46(20), 10359-10372.

Mdlongwa, P., Oluyede, B. O., Amey, A., \& Huang, S. (2017). The Burr XII modified Weibull distribution: model, properties and applications. Electronic J. of Applied Statistical Analysis, 10(1), 118-145.

Mead, M. E. (2014). An extended Pareto distribution. PJSOR, 10, 313-329.

Muhammad, M. (2016). A generalization of the BurrXII-Poisson distribution and its applications. J. Stat. Appl. Pro., 
$5(1), 29-41$.

Muhammad, M. (2017). The complementary exponentiated BurrXII Poisson distribution: model, properties and application. J. Stat. Appl. Pro. 6(1), 33-48.

Muhammad, M. (2017). A new lifetime model with a bounded support. ARJOM (Asian Research Journal of Mathematics), 7(3), 1-11.

Nadarajah, S., \& Li, R. (2017). Exact distribution of a modified Behrens-Fisher statistic. Commun. Statist. Simulation and Computation, 46(9), 6845-6864.

Najarzadegan, H., Alamatsaz, M. H., \& Hayati, S. Truncated Weibull-G more flexible and more reliable that beta-G distribution. IJSP, 6(5), online 1-17.

Nasir, M. A., Tahir, M. H., Jamal, F., \& Özel, G. (2017). A new generalized Burr family of distributions for the lifetime data. J. Stat. Appl. Pro., 6(2), 401-417.

Nofal, Z., Afify, A., Yousof, H., Dra, D. C. T. G., \& Louzada, F. (2017). The transmuted exponentiated additive Weibull distribution: properties and applications. J. Modern Applied Statistical Methods forthcoming.

Oguntunde, P. E., \& Adejumo, A. O. (2015). The generalized inverted generalized exponential distribution with an application to a censored data. J. Stat. Appl. Pro., 4(2), 223-230.

Okasha, H. M., El-Baz, A. H., Tarabia, A. M. K., \& Basheer, A. M. (2017). Extended inverse Weibull distribution with reliability application. Journal of th Egyptian Mathematical Society, 25, 343-349.

Oluyede, B. O., Motsewabagale, G., Huang, S., Warahena-Liyanage, G., \& Pararai, M. (2016). The Dagum-Poisson distribution: model, properties and application. Electronic J. of Applied Statistical Analysis, 9(1), 169-197.

Park, S., \& Park, J. (2018). A general class of flexible Weibull distributions, CSTM, 47(4), 767-778.

Rasekhi, M., Zayed, M., \& Yousof, H. M. (2017). A new two-parameter Weibull distribution with variable shapes for the hazard rate. J. Modern Applied Statistical methods (JMASM), 1-22.

Reyes, J., Vilca, F., Gallardo, D., \& Gómez, W. (2017). Modified slash Birnbaum-Saunders distribution, HJSM, 46(5), 969-984.

Roozegar, R., \& Esfandiary, F. (2015). The McDonald quasi Lindley distribution and its statistical properties and applications. J. Stat. Appl. Pro., 4(3), 375-386.

Saghir, A., Khadim, A., \& Lin, Z. (2016). The Maxwell length biased distribution: properties and Estimation. J. of Statistical Theory and Practice, 11, 26-40.

Sarhan, A. M. (2009). Generalized quadratic hazard rate distribution. International J. of Applied Math. E Stat., 14(S09), 94-106.

Sharma, V. K., Bakouch, H. S., \& Suthar, K. (2017). An extended Maxwell distribution: Properties and Applications. Commun. Statist. Simulation and Computation, 46(9), 6982-7007.

Singh, S. K., Singh, U., \& Sharma, V. K. (2014). The truncated Lindley distribution: Inference and Application. J. Stat. Appl. Pro., 3(2), 219-228.

Tahir, M., Alizadeh, M., Mansoor, M., Cordeiro, G. M., \& Zubair, M. (2014). The Weibull-power function distribution with applications. HJMS, Series B: Mathematics and Statistics.

Tahmasebi, S., \& Jafari, A. A. (2016). Generalized Gompertz-power series distribution. HJMS, 45(5), 1579-1604.

Yousof, H. M., Afify, A. Z., Hamedani, G. G., \& Aryal, G. (2016). The Burr X generator of distributions for lifetime data. JSTA, 16, 1-19.

Yousof, H. M., Afify, A. Z., Cordeiro, G. M., Alzaatreh, A., \& Ahsanullah, M. (December, 2017). A new four-parameter Weibull model for lifetime data. JSTA, 16(4), 448-466.

Zayed, M., \& Butt, N. S. (2017). The extended Fréchet distribution: Properties and Applications. Pak.j.stat.oper.res., $\operatorname{XIII}(3), 529-543$.

\section{Appendix A}

Theorem 1. Let $(\Omega, \mathcal{F}, \mathbf{P})$ be a given probability space and let $H=[a, b]$ be an interval for some $d<b(a=-\infty, b=\infty$ 
might as well be allowed). Let $X: \Omega \rightarrow H$ be a continuous random variable with the distribution function $F$ and let $q_{1}$ and $q_{2}$ be two real functions defined on $H$ such that

$$
\mathbf{E}\left[q_{2}(X) \mid X \geq x\right]=\mathbf{E}\left[q_{1}(X) \mid X \geq x\right] \xi(x), \quad x \in H,
$$

is defined with some real function $\eta$. Assume that $q_{1}, q_{2} \in C^{1}(H), \xi \in C^{2}(H)$ and $F$ is twice continuously differentiable and strictly monotone function on the set $H$. Finally, assume that the equation $\xi q_{1}=q_{2}$ has no real solution in the interior of $H$. Then $F$ is uniquely determined by the functions $q_{1}, q_{2}$ and $\xi$, particularly

$$
F(x)=\int_{a}^{x} C\left|\frac{\xi^{\prime}(u)}{\xi(u) q_{1}(u)-q_{2}(u)}\right| \exp (-s(u)) d u,
$$

where the function $s$ is a solution of the differential equation $s^{\prime}=\frac{\xi^{\prime} q_{1}}{\xi q_{1}-q_{2}}$ and $C$ is the normalization constant, such that $\int_{H} d F=1$.

\section{Copyrights}

Copyright for this article is retained by the author(s), with first publication rights granted to the journal.

This is an open-access article distributed under the terms and conditions of the Creative Commons Attribution license (http://creativecommons.org/licenses/by/4.0/). 